The Shifting Seasonal Mean Autoregressive Model and Seasonality in the Central England Monthly Temperature Series, 1772-2016

Changli He, Jian Kang, Timo Teräsvirta and Shuhua Zhang CREATES Research Paper 2018-15 


\title{
The Shifting Seasonal Mean Autoregressive Model and Seasonality in the Central England Monthly Temperature Series, $1772-2016$
}

\author{
Changli He*, Jian Kang*, Timo Teräsvirta ${ }^{\dagger \dagger}$ and Shuhua Zhang* \\ ${ }^{\dagger}$ CREATES, Aarhus University, Denmark \\ ${ }_{\ddagger}^{\ddagger}$ C.A.S.E., Humboldt-Universität zu Berlin, Germany \\ *Tianjin University of Finance and Economics, P.R. China
}

April 23, 2018

\begin{abstract}
In this paper we introduce an autoregressive model with seasonal dummy variables in which coefficients of seasonal dummies vary smoothly and deterministically over time. The error variance of the model is seasonally heteroskedastic and multiplicatively decomposed, the decomposition being similar to that in well known ARCH and GARCH models. This variance is also allowed to be smoothly and deterministically time-varying. Under regularity conditions, consistency and asymptotic normality of the maximum likelihood estimators of parameters of this model is proved. A test of constancy of the seasonal coefficients is derived. The test is generalised to specifying the parametric structure of the model. A test of constancy over time of the heteroskedastic error variance is presented. The purpose of building this model is to use it for describing changing seasonality in the wellknown monthly central England temperature series. More specifically, the idea is to find out in which way and by how much the monthly temperatures are varying over time during the period of more than 240 years, if they do. Misspecification tests are applied to the estimated model and the findings discussed.
\end{abstract}

Keywords: global warming; nonlinear time series; changing seasonality, smooth transition; testing constancy 
JEL Classification Codes: C22, C51, C52, Q54

Acknowledgements. Part of the work for this paper was carried out when the third author was visiting Tianjin University of Finance and Economics, whose kind hospitality is gratefully acknowledged. Material from this paper has been presented at the 2017 Conference on Econometric Models of Climate Change, Nuffield College, Oxford, September 2017, the workshop 'Advances in financial and time series econometrics', Kloster Drübeck, October 2017, the First Sydney Time Series and Forecasting Symposium, November 2017, the ANZESG meeting at the University of Queensland, Brisbane, February 2018, the 26th Annual Symposium of the Society for Nonlinear Dynamics and Econometrics, Keio University, Tokyo, March 2018, and seminars at the Bank of Estonia, Tallinn, City University of Hong Kong, and The University of Melbourne. We wish to thank participants, André Casalis, Rafael Kawka and Peter Phillips in particular, for their comments. Responsibility for any errors and shortcomings in this work remains ours.

\section{Introduction}

As a monthly temperature time series, the central England temperature (CET) series is quite unique because of its length. It extends over three and a half centuries and thus provides an opportunity to consider possible changes in the climate on a 'micro-level'. The series was originally compiled by Manley (1974) and covered the years 1659-1973; for a revised and extended series see Parker, Legg and Folland (1992). Several authors have studied properties of the series for various time periods. Harvey and Mills (2003) aggregated the series from 1723 to 1999 to the annual and seasonal level and considered deterministic trends using both local cubic trends and low-pass filters. Their conclusion was that within this period, no warming trend can be discerned. The main reason for this is that they found a rather strong downward movement in both the annual and seasonal series until around 1775, and the upward movement beginning thereafter only brought the temperatures to the level where they were in the beginning of the period. Vogelsang and Franses (2005) analysed the whole CET series from 1659 to 2000 using an autoregressive model augmented by a linear trend and a possible trend-break. Their conclusion was that there is a positive linear trend for months from October to April. This excludes the summer months. Recently, Proietti and Hillebrand (2017) used a structural time series model, separating the series from 1772 to 2013 into permanent and transitory components. The permanent component contained both a deterministic (linear) 
and a stochastic trend. They found that the deterministic trend is strongest for November, December and January, whereas the stochastic trend has the highest coefficients for April and May, and again for August, September and October.

This paper has two important purposes. The first one to develop a seasonal time series model which can adequately describe changes in seasonality over time in situations in which no single both observable and quantifiable cause for the change can be identified. The idea is to generalise the standard autoregressive model with seasonal dummy variables to the situation in which the seasonal pattern of the time series may not remain constant over time. The second purpose is to apply the model, called the Seasonal Shifting Mean Autoregressive (SSM-AR) model, to quantify potential warming in the monthly CET series. This implies a more detailed scrutiny of seasonality in this series than what is reported in hitherto published papers. The months in which warming, if any, has occurred will be found and its strength for each month estimated.

The plan of the paper is as follows. The SSM-AR model is introduced in Section 2. Its properties, such as the log-likelihood, score and the information matrix are presented in Section 3 and the Hessian in Section 4. Asymptotic theory for maximum likelihood estimators of parameters of the model is considered in Section 5. Specification and testing of the SSM-AR model is the topic of Section 6. Application to the CET series is described in Section 7. Section 8 contains discussion and final remarks. Proofs, estimated equations and some additional material can be found in Appendices.

\section{The model}

The number of nonlinear seasonal time series models is not large, but a few examples exist. Franses and de Bruin (2000) introduced a seasonal smooth transition autoregressive (SEASTAR) model and fitted it to seasonally unadjusted unemployment series. The purpose of the study was to study the effects of seasonal adjustments on the properties of these series. Ajmi, Ben Nasr and Boutahar (2008) generalised the model to the case where the variable to be explained is fractionally integrated and fitted the model to a quarterly US inflation series. van Dijk, Strikholm and Teräsvirta (2003) considered the time-varying smooth transition autoregressive (TV-STAR) model to investigate causes of changing seasonality in industrial output of G7 countries. Craig and Holt (2008) applied the TV-STAR model to studying effects of refrigeration on changes in seasonality in the US hog-corn price relationship using long historical time series; see also their study (Craig and Holt, 
2017) of the US egg market, 1890-2011.

As already mentioned, the SSM-AR model is a generalisation of an autoregressive model with seasonal dummy variables. In order to define the model, let $S$ be the length of the seasonal cycle (for example a year), and $j$ represent the $j$ th unit or 'season' (for example a month) within the cycle. Furthermore, $k$ indicates the $k$ th cycle, and $K$ is the total number of cycles, $k=0,1, \ldots, K-1$. The SSM-AR model for unit $s$ is defined as follows:

$$
y_{S k+s}=\sum_{j=1}^{S} \delta_{j}\left(\frac{S k+j}{S K}\right) D_{S k+s}^{(j)}+\sum_{i=1}^{p} \phi_{i} y_{S k+s-i}+\varepsilon_{S k+s}
$$

where $D_{S k+s}^{(j)}=1$ when $j=s$, zero otherwise $\left(D_{S k+s}^{(j)}\right.$ is the $s$ th seasonal dummy variable), $\varepsilon_{S k+s}$ is an error term with mean zero (more of it later) and the roots of $1-\sum_{i=1}^{p} \phi_{i} z^{i}=0$ lie outside the unit circle. The $j$ th time-varying coefficient $\delta_{j}\left(\frac{S k+j}{S K}\right)$ equals

$$
\delta_{j}\left(\frac{S k+j}{S K}\right)=\delta_{j 0}+\sum_{i=1}^{q_{j}} \delta_{j i} g_{j}\left(\frac{S k+j}{S K} ; \gamma_{j i}, \mathbf{c}_{j i}\right)
$$

where

$$
g_{j}\left(\frac{S k+j}{S K} ; \gamma_{j i}, c_{j i}\right)=\left(1+\exp \left\{-\gamma_{j i}\left(\frac{S k+j}{S K}-c_{j i}\right)\right\}\right)^{-1}
$$

or

$$
g_{j}\left(\frac{S k+j}{S K} ; \gamma_{j i}, c_{1 j i}, c_{2 j i}\right)=\left(1+\exp \left\{-\gamma_{j i}\left(\frac{S k+j}{S K}-c_{1 j i}\right)\left(\frac{S k+j}{S K}-c_{2 j i}\right)\right\}\right)^{-1}
$$

where $\gamma_{j i}>0$. It follows that (1) at time $S k+s$ may simply be written as

$$
y_{S k+s}=\delta_{s}\left(\frac{S k+s}{S K}\right)+\sum_{i=1}^{p} \phi_{i} y_{S k+s-i}+\varepsilon_{S k+s} .
$$

To fix notation, let $\boldsymbol{\theta}_{s}=\left(\boldsymbol{\delta}_{s}^{\prime}, \boldsymbol{\gamma}_{s}^{\prime}, \mathbf{c}_{s}^{\prime}\right)^{\prime}$, where $\boldsymbol{\delta}_{s}=\left(\delta_{s 0}, \delta_{s 1}, \ldots, \delta_{s q_{s}}\right)^{\prime}, \boldsymbol{\gamma}_{s}=$ $\left(\gamma_{s 1}, \ldots, \gamma_{s q_{s}}\right)^{\prime}$ and $\mathbf{c}_{s}=\left(c_{s 1}, \ldots, c_{s q_{s}}\right)^{\prime}$ are $q_{s} \times 1$ vectors. The autoregressive component has the parameter vector $\boldsymbol{\phi}=\left(\phi_{1}, \ldots, \phi_{p}\right)^{\prime}$. Furthermore, $\boldsymbol{\theta}_{M}=$ $\left(\boldsymbol{\theta}_{1}^{\prime}, \ldots, \boldsymbol{\theta}_{s}^{\prime}, \boldsymbol{\phi}^{\prime}\right)^{\prime} \in \Theta_{M}$ is a vector containing all parameters in the mean part of (1). Let $\boldsymbol{\theta}_{M}^{0}=\left(\boldsymbol{\theta}_{1}^{0 \prime}, \ldots, \boldsymbol{\theta}_{s}^{0 \prime}, \boldsymbol{\phi}^{0 \prime}\right)^{\prime}$ be the corresponding true parameter vector.

The error process $\left\{\varepsilon_{S k+s}\right\}$ is assumed to have time-varying variance. The error is decomposed as $\varepsilon_{S k+s}=z_{S k+s} \sigma_{s}\left(\frac{S k+s}{S K}\right)$, where $z_{S k+s} \sim \operatorname{iid} \mathcal{N}(0,1)$ and the deterministic time-varying component is defined as follows:

$$
\sigma_{s}^{2}\left(\frac{S k+j}{S K}\right)=\sum_{j=1}^{S} \sigma_{j}^{2}\left(\frac{S k+j}{S K}\right) D_{S k+s}^{(j)} .
$$


In $(6)$,

$$
\sigma_{j}^{2}\left(\frac{S k+j}{S K}\right)=\sigma_{j 0}^{2}+\sum_{i=1}^{r_{j}} \omega_{j i} g_{j}^{(v)}\left(\frac{S k+j}{S K} ; \gamma_{j i}^{(v)}, c_{j i}^{(v)}\right)
$$

with

$$
g_{j}^{(v)}\left(\frac{S k+j}{S K} ; \gamma_{j i}^{(v)}, c_{j i}^{(v)}\right)=\left(1+\exp \left\{-\gamma_{j i}^{(v)}\left(\frac{S k+j}{S K}-c_{j i}^{(v)}\right)\right\}\right)^{-1}
$$

or

$$
g_{j}^{(v)}\left(\frac{S k+j}{S K} ; \gamma_{j i}^{(v)}, c_{j i}^{(v)}\right)=\left(1+\exp \left\{-\gamma_{j i}^{(v)}\left(\frac{S k+j}{S K}-c_{1 j i}^{(v)}\right)\left(\frac{S k+j}{S K}-c_{2 j i}^{(v)}\right)\right\}\right)^{-1}
$$

where $\gamma_{j i}^{(v)}>0, i=1, \ldots, r_{j}$ and $j=1, \ldots, S$. To guarantee positivity of the variance, $\sigma_{j 0}^{2}>0$ and $\sigma_{j 0}^{2}+\sum_{i=1}^{k} \omega_{j i}>0$ for $k=1, \ldots, r_{j}$ and $j=1, \ldots, S$. This definition imposes restrictions on $\omega_{j i}, i=1, \ldots, r_{j}$. For the individual unit $s$ it conforms to the one in Silvennoinen and Teräsvirta (2016). A difference compared to that definition is that is $\sigma_{S k+s}^{2}$ is seasonal. Let $\boldsymbol{\theta}_{s}^{(v)}=\left(\sigma_{s 0}^{2}, \boldsymbol{\omega}_{s}^{\prime}, \boldsymbol{\gamma}_{s}^{(v) \prime}, \mathbf{c}_{s}^{(v) \prime}\right)^{\prime}$, where $\boldsymbol{\omega}_{s}=\left(\omega_{s 1}, \ldots, \omega_{s r_{s}}\right)^{\prime}, \boldsymbol{\gamma}_{s}^{(v)}=\left(\gamma_{s 1}, \ldots, \gamma_{s r_{s}}\right)^{\prime}$ and $\mathbf{c}_{s}^{(v)}=\left(c_{s 1}^{(v)}, \ldots, c_{s r_{s}}^{(v)}\right)^{\prime}$. Finally, let $\boldsymbol{\theta}_{V}=\left(\boldsymbol{\theta}_{1}^{(v) \prime}, \ldots, \boldsymbol{\theta}_{S}^{(v) \prime}\right)^{\prime} \in \Theta_{V}$ contain all parameters of (6) and denote the corresponding true parameter vector by $\boldsymbol{\theta}_{V}^{0}$

A special case of this SSM-AR model is the one in which $\sigma_{S k+s}^{2}=\sigma^{2}$, that is, the errors themselves are $\operatorname{iid}\left(0, \sigma^{2}\right)$. When $S=1$ and $\sigma_{S k+s}^{2}=\sigma^{2}$, the SSM-AR model collapses into the Shifting Mean Autoregressive (SM-AR) model by González and Teräsvirta (2008).

The SSM-AR model differs from the SEASTAR model in four respects. First, all units have a different transition function. Second, the transition variable is rescaled time. Third, the autoregressive part has constant coefficients, although this can be generalised. Finally, the error variance is not constant over time.

In order to define the seasonally shifting mean, let $\mathrm{L}$ be the lag operator: $\mathrm{L} x_{t}=x_{t-1}$, and write

$$
\phi(\mathrm{L})=1-\sum_{i=1}^{p} \phi_{i} \mathrm{~L}^{i} .
$$

Then (1) can be written as follows:

$$
y_{S k+s}=\phi^{-1}(\mathrm{~L})\left\{\sum_{j=1}^{S} \delta_{j}\left(\frac{S k+j}{S K}\right) D_{S k+s}^{(j)}+\varepsilon_{S k+s}\right\} .
$$


where $\phi^{-1}(\mathrm{~L})=\sum_{j=0}^{\infty} \psi_{j} z^{j}$ with $\psi_{0}=1$. For $j=s$, see (5),

$$
\begin{aligned}
y_{S k+s} & =\phi^{-1}(\mathrm{~L})\left\{\delta_{s}\left(\frac{S k+s}{S K}\right) D_{S k+s}^{(s)}+\varepsilon_{S k+s}\right\} \\
& =\sum_{i=0}^{\infty} \psi_{i}\left\{\delta_{s}\left(\frac{S k+s-i}{S K}\right) D_{S k+s-i}^{(s)}+\varepsilon_{S k+s-i}\right\} \\
& =\sum_{i=0}^{\infty} \psi_{i S} \delta_{s}\left(\frac{S(k-i)+s}{S K}\right) D_{S(k-i)+s}^{(s)}+\sum_{i=0}^{\infty} \psi_{i} \varepsilon_{S k+s-i}
\end{aligned}
$$

where $\phi^{-1}(\mathrm{~L})=\sum_{j=0}^{\infty} \psi_{j} z^{j}$ with $\psi_{0}=1$, and $\delta_{s}(x)=0$ for $x<0$. Furthermore, from (9), the shifting mean for unit $s$

$$
\mu_{S k+s}=\mathrm{E} y_{S k+s}=\phi^{-1}(1) \delta_{s}\left(\frac{S k+s}{S K}\right)+O\left(\frac{1}{S K}\right)
$$

assuming that the roots of $\phi(z)=0$ lie outside the unit circle and that $\sum_{i=0}^{\infty}\left|\psi_{i}\right|<\infty$. Under these conditions, as $y_{S k+s}-\mathrm{E} y_{S k+s}$ has mean zero, $y_{S k+s}$ may be called shifting-mean stationary. The last term in (11) that vanishes asymptotically is due to the fact that the model is nonlinear. ${ }^{1}$

It can be seen from equations (3) or (4) that the conditional mean component of the SSM-AR model is not identified if at least one element $\delta_{j}\left(\frac{S k+j}{S K}\right)$ is constant over time. Constancy may be achieved for example by setting or $\delta_{j i}=0$ in (2) or $\gamma_{j i}=0$ in (3) or (4) for $i=1, \ldots, q_{j}$. For this reason, it is necessary to test constancy of $\delta_{j}\left(\frac{S k+j}{S K}\right)$ for all $j=1, \ldots, S$ assuming heteroskedastic errors before fitting an SSM-AR model to the series. This testing problem is discussed in Section 6.2. Testing constancy of the error variance is discussed in Section 6.5, but a model with constant error variance has to be fitted to the data before the structure of the error variance can be considered.

If the SSM-AR model is identified and its parameters estimated, plotting

$$
\widehat{\mu}_{S k+s}=\widehat{\phi}^{-1}(1) \widehat{\delta}_{s}\left(\frac{S k+s}{S K}\right)
$$

$s=1, \ldots, S$, for $k=0,1, \ldots, K-1$, amounts to plotting the estimated systematic component (the seasonal mean) of the model unit by unit.

\footnotetext{
${ }^{1}$ We are grateful to Peter Phillips for pointing this out to us.
} 


\section{Log-likelihood, score and the information matrix}

Assuming independent errors with mean zero and time-varying variance $\sigma_{S k+s}^{2}$, the quasi log-likelihood function ( $S K$ observations) of the model for the seasonal unit $s$ is defined as follows:

$$
L_{S K}(\boldsymbol{\theta}, \boldsymbol{\varepsilon})=\mathrm{const}-\frac{1}{2} \sum_{k=0}^{K-1} \sum_{s=1}^{S} \ln \sigma_{S k+s}^{2}-\frac{1}{2} \sum_{k=0}^{K-1} \sum_{s=1}^{S} \frac{\varepsilon_{S k+s}^{2}}{\sigma_{S k+s}^{2}}
$$

where, from (1), $\varepsilon_{S k+s}=y_{S k+s}-\delta_{s}\left(\frac{S k+j}{S K}\right)-\sum_{i=1}^{p} \phi_{i} y_{S k+s-i}$, and $\sigma_{S k+s}^{2}$ is defined by (6).

For notational simplicity it is in this section assumed that $q_{j}=1$ in (2) and $r_{j}=1$ in (6) and that the transition function is defined by (3). Generalisations are straightforward. Consequently, write $\gamma_{j 1}=\gamma_{j}$ and $c_{j 1}=c_{j}$, and $\gamma_{j 1}^{(v)}=\gamma_{j}^{(v)}$ and $c_{j 1}^{(v)}=c_{j}^{(v)}$. Let now $j=s$. To further simplify the notation, write $g_{s}\left(\frac{S k+s}{S K}\right)=g_{s}$. The partial derivatives of $\delta_{s}\left(\frac{S k+s}{S K}\right)$ are given in the following lemma.

Lemma 1 The partial derivatives

$$
\frac{\partial \delta_{s}\left(\frac{S k+s}{S K}\right)}{\partial \boldsymbol{\theta}_{s}}=\left(\frac{\partial \delta_{s}\left(\frac{S k+s}{S K}\right)}{\partial \delta_{s 0}}, \frac{\partial \delta_{s}\left(\frac{S k+s}{S K}\right)}{\partial \delta_{s 1}}, \frac{\partial \delta_{s}\left(\frac{S k+s}{S K}\right)}{\partial \gamma_{s}}, \frac{\partial \delta_{s}\left(\frac{S k+s}{S K}\right)}{\partial c_{s}}\right)^{\prime}
$$

are as follows: $\partial \delta_{s}\left(\frac{S k+s}{S K}\right) / \partial \delta_{s 0}=1, \partial \delta_{s}\left(\frac{S k+s}{S K}\right) / \partial \delta_{s 1}=g_{s}$,

$$
\frac{\partial \delta_{s}\left(\frac{S k+s}{S K}\right)}{\partial \gamma_{s}}=-\delta_{s 1} g_{s}\left(1-g_{s}\right)\left(\frac{S k+s}{S K}-c_{s}\right)
$$

and

$$
\frac{\partial \delta_{s}\left(\frac{S k+s}{S K}\right)}{\partial c_{s}}=\delta_{s 1} \gamma_{s} g_{s}\left(1-g_{s}\right\}
$$

Furthermore, $\partial \delta_{s}\left(\frac{S k+s}{S K}\right) / \partial \boldsymbol{\theta}_{j}=\mathbf{0}$ for any $j \neq s$.

Now let $\boldsymbol{\theta}_{s}^{(v)}=\left(\sigma_{s 0}^{2}, \omega_{s 1}, \gamma_{s}^{(v)}, c_{s}^{(v)}\right)^{\prime}$ and write $g_{s 1}^{(v)}\left(\frac{S k+s}{S K}\right)=g_{s 1}^{(v)}$. The partial derivatives of $\sigma_{s}^{2}\left(\frac{S k+s}{S K}\right)$ are given in the following lemma.

Lemma 2 The partial derivatives

$$
\frac{\partial \sigma_{s}^{2}\left(\frac{S k+s}{S K}\right)}{\partial \boldsymbol{\theta}_{s}^{(v)}}=\left(\frac{\partial \sigma_{s}^{2}\left(\frac{S k+s}{S K}\right)}{\partial \sigma_{s 0}^{2}}, \frac{\partial \sigma_{s}^{2}\left(\frac{S k+s}{S K}\right)}{\partial \omega_{s 1}}, \frac{\partial \sigma_{s}^{2}\left(\frac{S k+s}{S K}\right)}{\partial \gamma_{s 1}^{(v)}}, \frac{\partial \sigma_{s}^{2}\left(\frac{S k+s}{S K}\right)}{\partial c_{s 1}^{(v)}}\right)^{\prime}
$$


are as follows: $\partial \sigma_{s}^{2}\left(\frac{S k+s}{S K}\right) / \partial \sigma_{s 0}^{2}=1, \partial \sigma_{s}^{2}\left(\frac{S k+s}{S K}\right) / \partial \omega_{s 1}=g_{s 1}^{(v)}$,

$$
\frac{\partial \sigma_{s}^{2}\left(\frac{S k+s}{S K}\right)}{\partial \gamma_{s 1}^{(v)}}=\omega_{s 1} g_{s 1}^{(v)}\left(1-g_{s 1}^{(v)}\right)\left(\frac{S k+s}{S K}-c_{s 1}^{(v)}\right)
$$

and

$$
\frac{\partial \sigma_{s}^{2}\left(\frac{S k+s}{S K}\right)}{\partial c_{s 1}^{(v)}}=-\gamma_{s 1}^{(v)} \omega_{s 1} g_{s 1}^{(v)}\left(1-g_{s 1}^{(v)}\right)
$$

See Amado and Teräsvirta (2013, Lemma A.1). As in the mean component, $\partial \sigma_{s}^{2}\left(\frac{S k+s}{S K}\right) / \partial \boldsymbol{\theta}_{j}^{(v)}=\mathbf{0}$ for $j \neq s$.

We can now define the average score of the mean parameter block of (12):

Lemma 3 The $(4 S+p) \times 1$ average score function $(1 / S K) \partial L_{S K}(\boldsymbol{\theta}, \boldsymbol{\varepsilon}) / \partial \boldsymbol{\theta}$ of the mean parameter block of (12) has the following form. The partial derivatives with respect to $\boldsymbol{\theta}_{s}$ and $\boldsymbol{\phi}$ equal

$$
\mathbf{s}_{K}\left(\boldsymbol{\theta}_{s}\right)=\frac{1}{K} \sum_{k=0}^{K-1} \frac{\varepsilon_{S k+s}}{\sigma_{s}^{2}\left(\frac{S k+s}{S K}\right)} \frac{\partial \delta_{s}\left(\frac{S k+s}{S K}\right)}{\partial \boldsymbol{\theta}_{s}}
$$

for $s=1, \ldots, S$, and

$$
\mathbf{s}_{S K}(\phi)=\frac{1}{S K} \sum_{k=0}^{K-1} \sum_{j=1}^{S} \frac{\varepsilon_{S k+j}}{\sigma_{s}^{2}\left(\frac{S k+j}{S K}\right)} \mathbf{y}_{S k+j-1}
$$

where $\mathbf{y}_{S k+j}=\left(y_{S k+j}, \ldots, y_{S k+j-p+1}\right)^{\prime}$. The elements of $\partial \delta_{s}\left(\frac{S k+s}{S K}\right) / \partial \boldsymbol{\theta}_{s}$ are defined in Lemma 1.

Likewise, the following lemma postulates the average score of the variance parameter block of (12).

Lemma 4 The sth $4 \times 1$ block of the average score function $(1 / S K) \partial L_{S K}\left(\boldsymbol{\theta}_{V}, \boldsymbol{\varepsilon}\right) / \partial \boldsymbol{\theta}_{V}$ of the variance part of (12) equals

$$
\mathbf{s}_{K}\left(\boldsymbol{\theta}_{s}^{(v)}\right)=\frac{1}{2 K} \sum_{k=0}^{K-1}\left(\frac{\varepsilon_{S k+s}^{2}}{\sigma_{s}^{2}\left(\frac{S k+s}{S K}\right)}-1\right) \frac{1}{\sigma_{s}^{2}\left(\frac{S k+s}{S K}\right)} \frac{\partial \sigma_{s}^{2}\left(\frac{S k+s}{S K}\right)}{\partial \boldsymbol{\theta}_{s}^{(v)}}
$$

$s=1, \ldots, S$, where the elements of $\partial \sigma_{s}^{2}\left(\frac{S k+s}{S K}\right) / \partial \boldsymbol{\theta}_{s}^{(v)}$ are defined in Lemma 2. 
Since the mean and variance components do not have common parameters, the information matrix $\mathbf{J}\left(\boldsymbol{\theta}^{0}\right)$ of the log-likelihood (12) has two large diagonal blocks, one for the mean and the other for the variance parameters. In the following, all partial derivatives are evaluated under $\boldsymbol{\theta}=\boldsymbol{\theta}^{0}$. The mean block of the information matrix is given in the following lemma:

Lemma 5 The mean block of the information matrix of the log-likelihood (12) has the form

$$
\begin{aligned}
\mathbf{J}^{M}\left(\boldsymbol{\theta}_{M}^{0}\right)= & \mathrm{E} \lim _{K \rightarrow \infty} \mathbf{s}_{S K}\left(\boldsymbol{\theta}_{M}^{0}\right) \mathbf{s}_{S K}^{\prime}\left(\boldsymbol{\theta}_{M}^{0}\right) \\
= & {\left[\begin{array}{ccccc}
\mathbf{J}^{M}\left(\boldsymbol{\theta}_{1}^{0}\right) & \mathbf{0} & \ldots & \mathbf{0} & \mathbf{J}^{M}\left(\boldsymbol{\theta}_{1}^{0}, \boldsymbol{\phi}^{0}\right) \\
& \mathbf{J}^{M}\left(\boldsymbol{\theta}_{2}^{0}\right) & \ldots & \mathbf{0} & \mathbf{J}^{M}\left(\boldsymbol{\theta}_{2}^{0}, \boldsymbol{\phi}^{0}\right) \\
& & \ldots & & \ldots \\
\mathbf{0} & \mathbf{0} & \ldots & \mathbf{J}^{M}\left(\boldsymbol{\theta}_{S}^{0}\right) & \mathbf{J}^{M}\left(\boldsymbol{\theta}_{S}^{0}, \boldsymbol{\phi}^{0}\right) \\
\mathbf{J}^{M}\left(\boldsymbol{\phi}^{0}, \boldsymbol{\theta}_{1}^{0}\right) & \mathbf{J}^{M}\left(\boldsymbol{\phi}^{0}, \boldsymbol{\theta}_{2}^{0}\right) & \ldots & \mathbf{J}^{M}\left(\boldsymbol{\phi}^{0}, \boldsymbol{\theta}_{S}^{0}\right) & \mathbf{J}^{M}\left(\boldsymbol{\phi}^{0}\right)
\end{array}\right] }
\end{aligned}
$$

Its nonzero blocks are

$$
\mathbf{J}^{M}\left(\boldsymbol{\theta}_{s}^{0}\right)=\int_{0}^{1} \frac{1}{\sigma_{s}^{2}(r)} \frac{\partial \delta_{s}(r)}{\partial \boldsymbol{\theta}_{s}} \frac{\partial \delta_{s}(r)}{\partial \boldsymbol{\theta}_{s}^{\prime}} d r
$$

$s=1, \ldots, S$,

$$
\mathbf{J}^{M}\left(\boldsymbol{\theta}_{s}^{0}, \phi^{0}\right)=\frac{1}{\phi(1)} \int_{0}^{1} \frac{1}{\sigma_{s}^{2}(r)} \frac{\partial \delta_{s}(r)}{\partial \boldsymbol{\theta}_{s}} \boldsymbol{\delta}_{s-1}^{\prime}(r) d r
$$

where $\boldsymbol{\delta}_{s}(r)=\left(\delta_{s}(r), \ldots, \delta_{s-p+1}(r)\right)^{\prime}, s=1, \ldots, S$, and $\mathbf{J}^{M}\left(\phi^{0}\right)$ whose diagonal elements equal

$$
\left[\mathbf{J}^{M}\left(\phi^{0}\right)\right]_{n n}=\sum_{i=0}^{\infty} \sum_{j=0}^{\infty} \psi_{i S} \psi_{j S} \frac{1}{S} \sum_{s=1}^{S} \int_{0}^{1} \frac{\delta_{s-n}(r)}{\sigma_{s}^{2}(r)} d r+\sum_{i=0}^{\infty} \psi_{i S}^{2}
$$

for $n=1, \ldots, p$. In addition, $\left[\mathbf{J}^{M}\left(\phi^{0}\right)\right]_{m n}=0$ for $m \neq n$. The elements of $\partial \delta_{s}(r) / \partial \boldsymbol{\theta}_{s}$ are defined in Lemma 1 .

Proof. See Appendix A.

The variance block of the information matrix is itself block diagonal, as the $S$ units of (6) do not share any parameters. The $s$ th diagonal block of this matrix is given by the lemma below.

Lemma 6 The sth diagonal block of the variance component of the information matrix of the log-likelihood (12) has the form

$$
\mathbf{J}^{V}\left(\boldsymbol{\theta}_{s}^{(v) 0}\right)=\frac{1}{2} \int_{0}^{1} \frac{1}{\sigma_{s}^{4}(r)} \frac{\partial \sigma_{s}^{2}(r)}{\partial \boldsymbol{\theta}_{s}^{(v)}} \frac{\partial \sigma_{s}^{2}(r)}{\partial \boldsymbol{\theta}_{s}^{(v) \prime}} d r
$$

$s=1, \ldots, S$. The elements of $\partial \sigma_{s}^{2}(r) / \partial \boldsymbol{\theta}_{s}^{(v)}$ are defined in Lemma 2. 
The whole information matrix is defined as $\mathbf{J}\left(\boldsymbol{\theta}^{0}\right)=\operatorname{diag}\left(\mathbf{J}^{M}\left(\boldsymbol{\theta}_{M}^{0}\right), \mathbf{J}^{V}\left(\boldsymbol{\theta}_{V}^{0}\right)\right)$.

\section{The Hessian}

If the errors are not assumed normal, the Hessian matrix of (12) is needed for statistical inference. It is block diagonal as the mean and the variance components do not have common parameters. The next two lemmas specify the nonzero blocks of this matrix.

Lemma 7 The average Hessian matrix for the mean component of the loglikelihood (12) equals

$$
\mathbf{H}_{S K}^{M}=\left[\begin{array}{cc}
\mathbf{H}_{\theta S K}^{M} & \mathbf{H}_{\theta \phi S K}^{M} \\
& \mathbf{H}_{\phi S K}^{M}
\end{array}\right]
$$

where $\mathbf{H}_{\theta_{s} K}^{M}=\operatorname{diag}\left(\mathbf{H}_{\theta_{1} K}^{M}, \ldots, \mathbf{H}_{\theta_{S} K}^{M}\right)$ with

$$
\mathbf{H}_{\theta_{s} K}^{M}=-\frac{1}{K} \sum_{k=0}^{K-1} \frac{1}{\sigma_{S k+s}^{2}}\left\{\frac{\partial \varepsilon_{S k+s}}{\partial \boldsymbol{\theta}_{s}} \frac{\partial \varepsilon_{S k+s}}{\partial \boldsymbol{\theta}_{s}^{\prime}}+\varepsilon_{S k+s} \frac{\partial^{2} \varepsilon_{S k+s}}{\partial \boldsymbol{\theta}_{s} \partial \boldsymbol{\theta}_{s}^{\prime}}\right\} .
$$

In (19),

$$
\frac{\partial^{2} \delta_{s}\left(\frac{S k+s}{S K}\right)}{\partial \boldsymbol{\theta}_{s} \partial \boldsymbol{\theta}_{s}^{\prime}}=\left[\begin{array}{ccc}
\frac{\partial^{2} \delta_{s}\left(\frac{S k+s}{S K}\right)}{\partial \delta_{s} \partial \boldsymbol{\delta}_{s}^{\prime}} & \frac{\partial^{2} \delta_{s}\left(\frac{S k+s}{S K}\right)}{\partial \boldsymbol{\delta}_{s} \partial \gamma_{s}} & \frac{\partial^{2} \delta_{s}\left(\frac{S k+s}{S K}\right)}{\partial \delta_{s} \partial c_{s}} \\
& \frac{\partial^{2} \delta_{s}\left(\frac{S k+s}{S K}\right)}{\partial \gamma_{s}^{2}} & \frac{\partial^{2} \delta_{s}\left(\frac{S k+s}{S K}\right)}{\partial \gamma_{s} \partial c_{s}} \\
& & \frac{\partial^{2} \delta_{s}\left(\frac{S k+s}{S K}\right)}{\partial c_{s}^{2}}
\end{array}\right]
$$

where $\partial^{2} \delta_{s}\left(\frac{S k+s}{S K}\right) / \partial \boldsymbol{\delta}_{s} \partial \boldsymbol{\delta}_{s}^{\prime}=\mathbf{0}$,

$$
\begin{gathered}
\frac{\partial^{2} \delta_{s}\left(\frac{S k+s}{S K}\right)}{\partial \boldsymbol{\delta}_{s} \partial \gamma_{s}}=\left(0, g_{s}\left(1-g_{s}\right)\left(\frac{S k+s}{S K}-c_{s}\right)\right)^{\prime} \\
\frac{\partial^{2} \delta_{s}\left(\frac{S k+s}{S K}\right)}{\partial \boldsymbol{\delta}_{s} \partial c_{s}}=\left(0,-\gamma_{s} g_{s}\left(1-g_{s}\right)\right)^{\prime} \\
\frac{\partial^{2} \delta_{s}\left(\frac{S k+s}{S K}\right)}{\partial \gamma_{s}^{2}}=\delta_{s 1} g_{s}\left(1-g_{s}\right)\left\{1-2 g_{s}\right)\left(\frac{S k+s}{S K}-c_{s}\right)^{2} \\
\frac{\partial^{2} \delta_{s}\left(\frac{S k+s}{S K}\right)}{\partial \gamma_{s} \partial c_{s}}=-\delta_{s 1} g_{s}\left(1-g_{s}\right)\left\{1+\gamma_{s}\left(1-2 g_{s}\right)\left(\frac{S k+s}{S K}-c_{s}\right)\right\}
\end{gathered}
$$

and

$$
\frac{\partial^{2} \delta_{s}\left(\frac{S k+s}{S K}\right)}{\partial c_{s}^{2}}=\delta_{s 1} \gamma_{s}^{2} g_{s}\left(1-g_{s}\right)\left(1-2 g_{s}\right)
$$


Furthermore, $\mathbf{H}_{\theta \phi S K}^{M}=\left(\mathbf{H}_{\theta_{1} \phi S K}^{M}, \ldots, \mathbf{H}_{\theta_{S} \phi S K}^{M}\right)^{\prime}$ where

$$
\mathbf{H}_{\theta_{s} \phi S K}^{M}=\frac{1}{K} \sum_{k=0}^{K-1} \frac{1}{\sigma_{S k+s}^{2}} \frac{\partial \delta_{s}\left(\frac{S k+s}{S K}\right)}{\partial \boldsymbol{\theta}_{s}} \mathbf{y}_{S k+s-1}^{\prime}
$$

and

$$
\mathbf{H}_{\phi S K}^{M}=\frac{1}{S K} \sum_{k=0}^{K-1} \sum_{s=1}^{S} \mathbf{y}_{S k+s-1} \mathbf{y}_{S k+s-1}^{\prime} .
$$

Lemma 8 The average Hessian matrix for the variance component of the log-likelihood (12) is diagonal and its sth diagonal block equals

$$
\begin{aligned}
\mathbf{H}_{\theta_{s}^{(v)} K}^{V}= & \frac{1}{2 K} \sum_{k=0}^{K-1}\left[\frac{\varepsilon_{t}^{2}}{\sigma_{S k+s}^{6}} \frac{\partial \sigma_{s k+s}^{2}}{\partial \theta_{s}^{(v)}} \frac{\partial \sigma_{s k+s}^{2}}{\partial \theta_{s}^{(v) \prime}}+\left(\frac{\varepsilon_{t}^{2}}{\sigma_{S k+s}^{2}}-1\right)\right. \\
& \left.\times \frac{1}{\sigma_{s k+s}^{2}}\left\{\frac{\partial \sigma_{S k+s}^{2}}{\partial \theta_{s}^{(v)}} \frac{\partial \sigma_{S k+s}^{2}}{\partial \theta_{s}^{(v) \prime}}+\frac{\partial^{2} \sigma_{S k+s}^{2}}{\partial \theta_{s}^{(v)} \partial \theta_{s}^{(v) \prime}}\right\}\right]
\end{aligned}
$$

where

$$
\frac{\partial^{2} \sigma_{s k+s}^{2}}{\partial \theta_{s}^{(v)} \partial \theta_{s}^{(v) \prime}}=\left[\begin{array}{cccc}
\frac{\partial^{2} \sigma_{S k+s}^{2}}{\partial\left(\sigma_{s 0}^{2}\right)^{2}} & \frac{\partial^{2} \sigma_{S k+s}^{2}}{\partial\left(\sigma_{s 0}^{2} \partial \omega_{s 1}\right.} & \frac{\partial^{2} \sigma_{S k+s}^{2}}{\partial\left(\sigma_{s 0}^{2}\right) \partial \gamma_{s}} & \frac{\partial^{2} \sigma_{S k+s}^{2}}{\partial\left(\sigma_{s}^{2}\right) \partial c_{s}} \\
& \frac{\partial^{2} \sigma_{S k+s}^{2}}{\partial \omega_{s 1}^{2}} & \frac{\partial^{2} \sigma_{S k+s}^{2}}{\partial \omega_{s 1} \partial \gamma_{s}} & \frac{\partial^{2} \sigma_{S k+s}^{2}}{\partial \omega_{s i s} \partial c_{s}} \\
& & \frac{\partial^{2} \sigma_{S k+s}^{2}}{\partial \gamma_{s}^{2}} & \frac{\partial^{2} \sigma_{S k+s}^{2}}{\partial \gamma_{s}^{2} \partial c_{s}} \\
& & & \frac{\partial^{2} \sigma_{S k+s}^{2}}{\partial c_{s}^{2}}
\end{array}\right]
$$

with $\partial^{2} \sigma_{S k+s}^{2} / \partial\left(\sigma_{S 0}^{2}\right)^{2}=\partial^{2} \sigma_{S k+s}^{2} /\left\{\partial\left(\sigma_{S 0}^{2}\right) \partial \omega_{s 1}\right\}=\partial^{2} \sigma_{S k+s}^{2} /\left\{\partial\left(\sigma_{S 0}^{2}\right) \partial \gamma_{s}\right\}=$ $\partial^{2} \sigma_{S k+s}^{2} /\left\{\partial\left(\sigma_{S 0}^{2}\right) \partial c_{s}\right\}=\partial^{2} \sigma_{S k+s}^{2} / \partial \omega_{s 1}^{2}=0$,

$$
\begin{gathered}
\frac{\partial^{2} \sigma_{S k+s}^{2}}{\partial \omega_{s 1} \partial \gamma_{s}}=g_{s 1}^{(v)}\left(1-g_{s 1}^{(v)}\right)\left(\frac{S k+s}{S K}-c_{s 1}^{(v)}\right) \\
\frac{\partial^{2} \sigma_{S k+s}^{2}}{\partial \omega_{s 1} \partial c_{s}}=-\gamma_{s 1}^{(v)} g_{s 1}^{(v)}\left(1-g_{s 1}^{(v)}\right) \\
\frac{\partial^{2} \sigma_{S k+s}^{2}}{\partial \gamma_{s}^{2}}=\omega_{s 1} g\left(1-g_{s 1}^{(v)}\right)\left(1-2 g_{s 1}^{(v)}\right)\left(\frac{S k+s}{S K}-c_{s 1}^{(v)}\right)^{2} \\
\frac{\partial^{2} \sigma_{S k+s}^{2}}{\partial \gamma_{s} \partial c_{s}}=-\omega_{s 1} g_{s 1}^{(v)}\left(1-g_{s 1}^{(v)}\right)\left\{1+\gamma_{s 1}^{(v)}\left(1-2 g_{s 1}^{(v)}\right)\left(\frac{S k+s}{S K}-c_{s 1}^{(v)}\right)\right\}
\end{gathered}
$$

and

$$
\frac{\partial^{2} \sigma_{S k+s}^{2}}{\partial c_{s}^{2}}=\left(\gamma_{s 1}^{(v)}\right)^{2} \omega_{s 1} g_{s 1}^{(v)}\left(1-g_{s 1}^{(v)}\right)\left(1-2 g_{s 1}^{(v)}\right)
$$




\section{$5 \quad$ Estimation of parameters}

\subsection{Zig-zag algorithm}

It follows from Lemmas 1 and 2 that the log-likelihood function is continuous. In addition, the mean and the variance components of the model do not have common parameters. Maximisation of the log-likelihood can therefore be carried by splitting each iteration into two components as Sargan (1964) suggested. The parameters in the mean part are estimated first, and parameters in the error variance thereafter, conditionally on the estimates of the mean parameters. Next the mean parameters are re-estimated conditionally on the estimated time-varying variances, followed by re-estimation of the variances. Sargan showed that under certain conditions this procedure of maximising a function with these two sets of parameters converges to a maximum. Oberhofer and Kmenta (1974) discussed the same algorithm in the log-likelihood context and called it the zig-zag algorithm. They demonstrated that the algorithm converges to at least a local maximum of the log-likelihood.

Both steps of the zig-zag algorithm are nonlinear. Finding initial values for the conditional mean may appear a difficult problem whenever $S>1$. However, because the dummy variables are orthogonal, the problem can be broken down to using two- or three-dimensional mini-grids for the slope and location parameters in the $S$ transition functions, depending the choice between (3) and (4). The 'linear' parameters, including the autoregressive coefficients, are obtained by simple regression conditionally on the relevant grid point. The combination of estimates minimising the sum of squared residuals from these regressions provides the starting-point for subsequent numerical maximisation of the log-likelihood (12). Orthogonality of the dummies also simplifies solving the nonlinear optimisation problem that is conveniently done by nonlinear least squares.

Estimation of the error variance is carried out unit by unit. It is advisable to begin each estimation from a set of starting-values to find at least a reasonable local optimum of the log-likelihood. Asymptotic properties of the maximum likelihood estimators of the parameters of the model are considered in the next section.

\subsection{Consistency and asymptotic normality}

In order to study asymptotic properties of the aforementioned maximum likelihood estimators we make the following assumptions about the model (1): 
A1 In the transition function $g_{s}\left(\frac{S k+s}{S K} ; \gamma_{s i}, c_{s i}\right), \gamma_{s i}>0, i=1, \ldots, q_{s}$; $c_{s 1}<\ldots<c_{s q_{s}}$. This implies $g_{s}\left(\frac{S k+s}{S K} ; \gamma_{s i}, c_{s i}\right) \neq g_{s}\left(\frac{S k+s}{S K} ; \gamma_{s j}, c_{s j}\right)$ for $i \neq j$. In addition, $\delta_{s i} \neq 0, i=1, \ldots, q_{s}$.

A2 In the transition function $g_{s}^{(v)}\left(\frac{S k+s}{S K} ; \gamma_{s i}^{(v)}, c_{s i}^{(v)}\right), \gamma_{s i}^{(v)}>0, i=1, \ldots, r_{s}$; $c_{s 1}^{(v)}<\ldots<c_{s r_{s}}^{(v)}$. This implies $g_{s}\left(\frac{S k+s}{S K} ; \gamma_{s i}, c_{s i}\right) \neq g_{s}\left(\frac{S k+s}{S K} ; \gamma_{s j}, c_{s j}\right)$ for $i \neq j$. In addition, $\omega_{s i} \neq 0, i=1, \ldots, r_{s}$.

A3 Parameter space $\Theta=\Theta_{M} \cup \Theta_{V}, \Theta_{M} \cap \Theta_{V}=\varnothing$, is compact, $\boldsymbol{\theta}^{0}$ is an interior point of $\Theta$.

A4 The density is positive (bounded away from zero) for all $\boldsymbol{\theta} \in \Theta$.

A5 The errors $z_{S k+s}=\varepsilon_{S k+s} / \sigma_{s}\left(\frac{S k+s}{S K}\right)$ are iid $\mathcal{N}(0,1)$.

A6 The roots of $\phi(z)=1-\sum_{i=1}^{p} \phi_{i} z^{i}=0$ lie outside the unit circle, and $\sum_{i=0}^{\infty}\left|\psi_{i}\right|<\infty$

If A1 is relaxed such that $c_{s j}=c_{s, j+1}$ for some $j$, then $g_{s}\left(\frac{S k+s}{S K} ; \gamma_{s j}, c_{s j}\right) \neq$ $g_{s}\left(\frac{S k+s}{S K} ; \gamma_{s, j+1}, c_{s, j+1}\right)$ requires $\gamma_{s j} \neq \gamma_{s, j+1}$. A similar argument is valid for A2. These assumptions, together with $\delta_{s i} \neq 0, i=1, \ldots, q_{s}$ and $\omega_{s i} \neq 0$, $i=1, \ldots, r_{s}, s=1, \ldots, S$, identify the model. Normality of errors may also be relaxed and replaced by $z_{S k+s} \sim \operatorname{iid}(0,1)$.

Consistency of the maximum likelihood estimators of the SSM-AR model is established by proving the following result:

Theorem 9 Consider the SSM-AR model (1) and suppose that Assumptions A1-A6 hold. Then the maximum likelihood estimator $\widehat{\boldsymbol{\theta}}$ is consistent for $\boldsymbol{\theta}^{0}$.

Proof. See Appendix A.

In order to consider asymptotic normality of $\widehat{\boldsymbol{\theta}}$ we make another assumption:

A7 Parameter vector $\boldsymbol{\theta}^{0}$ is an interior point of $\Theta$.

Establishing asymptotic normality of the maximum likelihood estimators of the parameters of the SSM-AR model requires proving the following result:

Theorem 10 Assume that the result of Theorem 9 holds, that is, the maximum likelihood estimator $\widehat{\boldsymbol{\theta}} \stackrel{p}{\rightarrow} \boldsymbol{\theta}^{0}$ as $K \rightarrow \infty$. Assume further that Assumptions A1-A7 are valid. Then

$$
\sqrt{K}\left(\widehat{\boldsymbol{\theta}}-\boldsymbol{\theta}^{0}\right) \stackrel{d}{\rightarrow} N\left(\mathbf{0}, \mathbf{J}^{-1}\left(\boldsymbol{\theta}^{0}\right)\right)
$$

as $K \rightarrow \infty$, where the information matrix $\mathbf{J}\left(\boldsymbol{\theta}^{0}\right)=\operatorname{diag}\left(\mathbf{J}^{M}\left(\boldsymbol{\theta}_{M}^{0}\right), \mathbf{J}^{V}\left(\boldsymbol{\theta}_{V}^{0}\right)\right)$ is defined in Lemmas 5 and 6.

Proof. See Appendix A. 


\section{Building SSM-AR models}

\subsection{General}

As is clear from Section 2, before an SSM-AR model can be estimated its form has to be specified. The number of transitions has to be determined from the data because typically there is little or no theory available to help the model builder to make the correct decision. Overestimating this number leads to difficulties because a model containing too many transitions either in the mean or the variance or both is not identified. The parameters of such a model cannot be estimated consistently. This is why one has to proceed from specific to general, which means testing each model against the next larger alternative. This reduces the risk of estimating unidentified models which can be big if the number of transitions is determined using model selection criteria such as AIC or BIC. The form of the mean is determined first, followed by that of the error variance.

After the model has been estimated as discussed in Section 5, it has to be evaluated. This means that the estimated model has to be subjected to misspecification tests to find out whether or not the model is adequate. Such tests are considered in Section 6.2.

\subsection{Testing constancy of coefficients of seasonal dum- mies}

\subsubsection{Joint test for all seasonal coefficients}

The first step in specifying an SSM-AR model is to test stability of the coefficients of its dummy variables. The null model is the standard autoregressive model with seasonally varying means:

$$
y_{S k+s}=\sum_{j=1}^{S} \delta_{j 0} D_{S k+s}^{(j)}+\sum_{i=1}^{p} \phi_{i} y_{S k+s-i}+\varepsilon_{S k+s}
$$

whereas the alternative is the SSM-AR model (1). Since the SSM-AR model is not identified when at least one coefficient $\delta_{j}\left(\frac{S k+j}{S K}\right)=\delta_{j 0}$, standard asymptotic inference in testing $\mathrm{H}_{0}: \delta_{j}\left(\frac{S k+j}{S K}\right)=\delta_{j 0}, j=1, \ldots, S$, is invalid, for discussion see, for example, Teräsvirta, Tjøstheim and Granger (2010, Chapter 5) and references therein, including Hansen (1996). This problem and solutions to it were first discussed by Davies (1977).

In order to test constancy against (at least) one transition in $\delta_{j}\left(\frac{S k+j}{S K}\right)$, our null hypothesis is $\mathrm{H}_{0}: \gamma_{j}=0, j=1, \ldots, S$, so $\delta_{j 1}$, and $c_{j 1}$ or $c_{j 1}$ and $c_{j 2}$, 
are unidentified nuisance parameters when this hypothesis holds. In order to circumvent the identification problem we follow Luukkonen, Saikkonen and Teräsvirta (1988) and expand $\left(1+\exp \left\{-\gamma_{j}\left(\frac{S k+j}{S K}-c_{j}\right)\right\}\right)^{-1}$ into a Taylor series around $\gamma_{j}=0$ and reparameterise (3) (or (4)) accordingly. Assuming $\delta_{j 1} \neq 0, j=1, \ldots, S$, and choosing the third-order polynomial expansion one obtains

$$
\delta_{j}\left(\frac{S k+j}{S K}\right)=\alpha_{j 0}+\alpha_{j 1} \frac{S k+j}{S K}+\alpha_{j 2}\left(\frac{S k+j}{S K}\right)^{2}+\alpha_{j 3}\left(\frac{S k+j}{S K}\right)^{3}+R_{3, S k+j}
$$

where $R_{3, S k+j}$ is the remainder. Since $\alpha_{j i}=\gamma_{j}^{i} \widetilde{\delta}_{j i}$, where $\widetilde{\delta}_{j i} \neq 0, i=1,2,3$, the resulting auxiliary SSM-AR model has the following form:

$$
\begin{aligned}
y_{S k+s}= & \sum_{j=1}^{S}\left\{\alpha_{j 0}+\alpha_{j 1} \frac{S k+j}{S K}+\alpha_{j 2}\left(\frac{S k+j}{S K}\right)^{2}+\alpha_{j 3}\left(\frac{S k+j}{S K}\right)^{3}\right\} D_{S k+s}^{(j)} \\
& +\sum_{i=1}^{p} \phi_{i} y_{S k+s-i}+\varepsilon_{S k+s}^{*}
\end{aligned}
$$

where $\varepsilon_{S k+s}^{*}=\varepsilon_{S k+s}+R_{3, S k+s}$, and the new null hypothesis equals $\mathrm{H}_{0}^{\prime}$ : $\boldsymbol{\alpha}_{1}=\ldots=\boldsymbol{\alpha}_{S}=\mathbf{0}$, where $\boldsymbol{\alpha}_{j}=\left(\alpha_{j 1}, \alpha_{j 2}, \alpha_{j 3}\right)^{\prime}$ for $j=1, \ldots, S$. Under this hypothesis, $R_{3, S k+s}=0$, and because we are considering a Lagrange multiplier test, the remainder does not affect the inference.

In order to derive the test statistic, it is assumed that $\sigma_{S k+s}^{2}=\sigma^{2}$ because the mean has to be specified before the error variance. The log-likelihood of (21), assuming normal errors and setting $R_{3, S k+s}=0$, becomes

$$
\begin{aligned}
L_{S K}^{A}(\boldsymbol{\theta}, \boldsymbol{\varepsilon})= & c-\frac{S K}{2} \ln \sigma^{2}-\frac{1}{2 S K} \sum_{k=0}^{K-1} \sum_{j=1}^{S} \frac{\varepsilon_{S k+j}^{2}}{\sigma^{2}} \\
= & c-\frac{S K}{2} \ln \sigma^{2}-\frac{1}{2 \sigma^{2} S} \sum_{j=1}^{S} \frac{1}{K} \sum_{k=0}^{K-1}\left(y_{S k+j}\right. \\
& -\left\{\alpha_{j 0}+\alpha_{j 1} \frac{S k+j}{S K}+\alpha_{j 2}\left(\frac{S k+j}{S K}\right)^{2}\right. \\
& \left.\left.+\alpha_{j 3}\left(\frac{S k+j}{S K}\right)^{3}\right\} D_{S k+s}^{(j)}-\sum_{i=1}^{p} \phi_{i} y_{S k+s-i}\right)^{2} .
\end{aligned}
$$

Let $\boldsymbol{\alpha}=\left(\boldsymbol{\alpha}_{1}^{\prime}, \ldots, \boldsymbol{\alpha}_{S}^{\prime}\right)^{\prime}, \boldsymbol{\alpha}_{0}=\left(\alpha_{10}, \ldots, \alpha_{S 0}\right)^{\prime}$, and $\boldsymbol{\phi}=\left(\phi_{1}, \ldots, \phi_{p}\right)^{\prime}$ as before. The blocks of the auxiliary average score

$$
\mathbf{s}_{K}\left(\boldsymbol{\alpha}, \boldsymbol{\alpha}_{0}, \boldsymbol{\phi}\right)=\left(\mathbf{s}_{K}^{\prime}\left(\boldsymbol{\alpha}_{1}\right), \ldots, \mathbf{s}_{K}^{\prime}\left(\boldsymbol{\alpha}_{S}\right), \mathbf{s}_{K}^{\prime}\left(\boldsymbol{\alpha}_{0}\right), \mathbf{s}_{K}^{\prime}(\boldsymbol{\phi})\right)^{\prime}
$$


are

$$
\mathbf{s}_{K}\left(\boldsymbol{\alpha}_{s}\right)=\frac{1}{\sigma^{2} K} \sum_{k=0}^{K-1} \varepsilon_{S k+s} \mathbf{k}_{S k+s}
$$

$s=1, \ldots, S$, where $\mathbf{k}_{S k+s}=\left(\frac{S k+s}{S K},\left(\frac{S k+s}{S K}\right)^{2},\left(\frac{S k+s}{S K}\right)^{3}\right)^{\prime}$, and

$$
\mathbf{s}_{K}\left(\boldsymbol{\alpha}_{0}\right)=\left(s_{K}\left(\alpha_{10}\right), \ldots, s_{K}\left(\alpha_{S 0}\right)\right)^{\prime}
$$

where

$$
\mathbf{s}_{K}\left(\alpha_{s 0}\right)=\frac{1}{\sigma^{2} K} \sum_{k=0}^{K-1} \varepsilon_{S k+s}
$$

$s=1, \ldots, S$. Finally, $\mathbf{s}_{S K}(\phi)$ is given in (14). We can now formulate the information matrix of the auxiliary log-likelihood (22).

Lemma 11 The information matrix of the auxiliary log-likelihood (22) under $H_{0}^{\prime}: \boldsymbol{\alpha}_{1}=\ldots=\boldsymbol{\alpha}_{S}=\mathbf{0}$ equals

$$
\begin{aligned}
\mathbf{J}^{A}\left(\boldsymbol{\alpha}, \boldsymbol{\alpha}_{0}, \boldsymbol{\phi}\right) & =\mathrm{E} \lim _{K \rightarrow \infty} \mathbf{s}_{S K}\left(\boldsymbol{\alpha}, \boldsymbol{\alpha}_{0}, \boldsymbol{\phi}\right) \mathbf{s}_{S K}^{\prime}(\boldsymbol{\alpha}, \boldsymbol{\alpha}, \boldsymbol{\phi}) \\
& =\left[\begin{array}{ccc}
\mathbf{J}^{A}(\boldsymbol{\alpha}) & \mathbf{J}^{A}\left(\boldsymbol{\alpha}, \boldsymbol{\alpha}_{0}\right) & \mathbf{J}^{A}(\boldsymbol{\alpha}, \boldsymbol{\phi}) \\
& \mathbf{I}_{S} & \mathbf{J}^{A}(\boldsymbol{\alpha}, \boldsymbol{\phi}) \\
& & \mathbf{J}^{A}(\boldsymbol{\phi})
\end{array}\right]
\end{aligned}
$$

where the $3 S \times 3 S$ matrix

$$
\mathbf{J}^{A}(\boldsymbol{\alpha})=\operatorname{diag}\left(\mathbf{J}^{A}\left(\boldsymbol{\alpha}_{1}\right), \ldots, \mathbf{J}^{A}\left(\boldsymbol{\alpha}_{S}\right)\right)
$$

with

$$
\mathbf{J}^{A}\left(\boldsymbol{\alpha}_{s}\right)=\frac{1}{\sigma^{2}} \int_{0}^{1} \mathbf{r r}^{\prime} d r=\frac{1}{\sigma^{2}}\left[\begin{array}{lll}
1 / 3 & 1 / 4 & 1 / 5 \\
& 1 / 5 & 1 / 6 \\
& & 1 / 7
\end{array}\right]
$$

for $s=1, \ldots, S$,

$$
\mathbf{J}^{A}\left(\boldsymbol{\alpha}, \boldsymbol{\alpha}_{0}\right)=\left[\begin{array}{c}
\mathbf{J}^{A}\left(\boldsymbol{\alpha}_{1}, \alpha_{10}\right) \\
\cdots \\
\mathbf{J}^{A}\left(\boldsymbol{\alpha}_{S}, \alpha_{S 0}\right)
\end{array}\right]
$$

where the $3 \times 1$ vector

$$
\mathbf{J}^{A}\left(\boldsymbol{\alpha}_{s}, \alpha_{s 0}\right)=\frac{1}{\sigma^{2}} \int_{0}^{1} \mathbf{r} d r=\frac{1}{\sigma^{2}}\left(\frac{1}{2}, \frac{1}{3}, \frac{1}{4}\right)^{\prime}
$$

for $s=1, \ldots, S$,

$$
\mathbf{J}^{A}\left(\alpha_{s 0}, \boldsymbol{\phi}\right)=\frac{1}{\sigma^{2} \phi(1)} \int_{0}^{1} \boldsymbol{\delta}_{s-1}^{\prime}(r) d r
$$


and

$$
\mathbf{J}^{A}(\boldsymbol{\alpha}, \boldsymbol{\phi})=\left[\begin{array}{c}
\mathbf{J}^{A}\left(\boldsymbol{\alpha}_{1}, \boldsymbol{\phi}\right) \\
\ldots \\
\mathbf{J}^{A}\left(\boldsymbol{\alpha}_{S}, \boldsymbol{\phi}\right)
\end{array}\right]
$$

where the $3 \times p$ matrix

$$
\mathbf{J}^{A}\left(\boldsymbol{\alpha}_{s}, \boldsymbol{\phi}\right)=\frac{1}{\sigma^{2} \phi(1)} \int_{0}^{1} \mathbf{r} \boldsymbol{\delta}_{s-1}^{\prime}(r) d r
$$

for $s=1, \ldots, S$, and, finally, $\left[\mathbf{J}^{A}(\phi)\right]_{m n}=[\mathbf{J}(\phi)]_{m n}$ assuming $\sigma_{s}^{2}(r)=\sigma^{2}$, $s=1, \ldots, S$, in $(18)$.

Proof. See Appendix A.

When Theorem 9 is valid, one can construct a test of the auxiliary null hypothesis $\mathrm{H}_{0}^{\prime}$ and thus also of the original one. The test statistic obtained by using the Lagrange multiplier or score principle is

$$
\begin{aligned}
L M(\boldsymbol{\theta})= & K\left\{\frac{1}{\sigma^{2} K} \sum_{k=0}^{K-1}\left(\widehat{\varepsilon}_{S k+1} \mathbf{k}_{S k+1}^{\prime}, \ldots, \widehat{\varepsilon}_{S(k+1)} \mathbf{k}_{S(k+1)}^{\prime}\right)^{\prime}\right\} \\
& \times\left\{\mathbf{J}^{A}(\boldsymbol{\alpha})-\left(\mathbf{J}^{A}\left(\boldsymbol{\alpha}, \boldsymbol{\alpha}_{0}\right), \mathbf{J}^{A}(\boldsymbol{\alpha}, \boldsymbol{\phi})\right)\right. \\
& \left.\times\left[\begin{array}{cc}
\mathbf{I}_{S} & \mathbf{J}^{A}\left(\boldsymbol{\alpha}_{0}, \boldsymbol{\phi}\right) \\
\mathbf{J}^{A}(\boldsymbol{\phi})
\end{array}\right]^{-1}\left[\begin{array}{c}
\mathbf{J}^{A}\left(\boldsymbol{\alpha}_{0}, \boldsymbol{\alpha}\right) \\
\mathbf{J}^{A}(\boldsymbol{\phi}, \boldsymbol{\alpha})
\end{array}\right]\right\}^{-1} \\
& \times\left\{\frac{1}{\sigma^{2} K} \sum_{k=0}^{K-1}\left(\widehat{\varepsilon}_{S k+1} \mathbf{k}_{S k+1}^{\prime}, \ldots, \widehat{\varepsilon}_{S(k+1)} \mathbf{k}_{S(k+1)}^{\prime}\right)\right\}
\end{aligned}
$$

where the residuals $\widehat{\varepsilon}_{S k+j}$ come from the estimated null model. The statistic (26) has an asymptotic $\chi^{2}$-distribution with $3 S$ degrees of freedom when the null hypothesis holds. To make (26) operational, the blocks of the information matrix have to be replaced by their consistent estimators.

In our case, the assumption of constant error variance is not valid. As the conditional mean is specified before the conditional variance, the form of $\sigma_{j}^{2}\left(\frac{S k+j}{S K}\right)$ is at that stage not known. Consequently, the statistic has to be robustified against heteroskedasticity. Following Wooldridge (1990), the robust statistic is conveniently computed in the ' $T R^{2}$ form' in three steps:

1. Estimate the null model, the SSM-AR model with constant coefficients on the dummy variables, and collect the residuals $\widehat{\varepsilon}_{S k+j}, k=$ $0,1, \ldots, K-1 ; j=1, \ldots, S$.

2. Regress 1 on $\widehat{\varepsilon}_{S k+j}, \widehat{\varepsilon}_{S k+j} \mathbf{y}_{S k+j-1}$ and $\widehat{\varepsilon}_{S k+j} \mathbf{k}_{S k+j}, j=1, \ldots, S$, and calculate the residual sum of squared errors $S S R$. 
3. Compute the test statistic (the uncentred coefficient of determination)

$$
L M=S K-S S R .
$$

Under $\mathrm{H}_{0}$, the robust statistic (27) has the same asymptotic null distribution as the nonrobust statistic (26).

While this test is suitable for our purposes, it may be noted that it is not an omnibus test. For a relevant omnibus test based on polynomials, see Baek, Cho and Phillips (2015).

\subsection{Testing constancy of subsets of coefficients}

If $\mathrm{H}_{0}^{\prime}$ and, consequently, $\mathrm{H}_{0}$ is rejected, it is of interest to know whether there exist seasonal components for which the seasonal coefficient $\delta_{j}\left(\frac{S k+j}{S K}\right)$ is nevertheless stable. This possibility can be studied by testing subhypotheses in (21). For example, it is possible to test $\mathrm{H}_{0 s}^{\prime}: \boldsymbol{\alpha}_{s}=\mathbf{0}$ for a single $s \in$ $\{1, \ldots, S\}$. The test is a special case of the previous one. The robust test is carried out as follows:

1. Estimate the null model, the auxiliary SSM-AR model with a constant ('zero order') coefficient on the dummy variables except the sth one, and collect the residuals $\widetilde{\varepsilon}_{S k+j}, k=0,1, \ldots, K-1 ; j=1, \ldots, S$.

2. Regress 1 on $\widetilde{\varepsilon}_{S k+j}, \widetilde{\varepsilon}_{S k+j} \mathbf{y}_{S k+j-1}$ and $\widetilde{\varepsilon}_{S k+j} \mathbf{k}_{S k+s}$ and calculate the residual sum of squared errors $S S R_{s}$.

3. Compute the test statistic (the uncentred coefficient of determination)

$$
L M_{s}=S K-S S R_{s} .
$$

Under $\mathrm{H}_{0}$, the statistic (28) has an asymptotic $\chi^{2}$-distribution with three degrees of freedom.

\subsection{Determining the number of transitions in the time- varying intercept}

If constancy of the coefficient of $D_{S k+j}^{(s)}$ is rejected, the next step is to test one transition against two. More generally, the problem is testing $q_{s}$ transitions against $q_{s}+1$. Here it is assumed that $q_{s}=1$. Under the alternative, the time-varying coefficient of $D_{S k+j}^{(s)}$ equals

$$
\delta_{s}\left(\frac{S k+s}{S K}\right)=\delta_{s 0}+\delta_{s 1} g_{s}\left(\frac{S k+s}{S K} ; \gamma_{s 1}, c_{s 1}\right)+\delta_{s 2} g_{s}\left(\frac{S k+s}{S K} ; \gamma_{s 2}, c_{s 2}\right) .
$$


The null hypothesis is chosen to be $\gamma_{s 2}=0$, so that under $\mathrm{H}_{0}, g\left(\frac{S k+s}{S K} ; \gamma_{q 2}, c_{q 2}\right)=$ $1 / 2$. The identification problem is solved as before: expand the transition function $g\left(\frac{S k+s}{S K} ; \gamma_{s 2}, c_{s 2}\right)$ into a Taylor series around $\gamma_{s 2}=0$ and reparameterise (29) accordingly. Assuming $\delta_{s 2} \neq 0$, this gives the following third-order polynomial expression for (29):

$$
\begin{aligned}
\delta_{s}^{A}\left(\frac{S k+j}{S K}\right)= & \alpha_{s 0}+\delta_{s 1} g\left(\frac{S k+s}{S K} ; \gamma_{s 1}, c_{s 1}\right)+\alpha_{s 1} \frac{S k+s}{S K}+\alpha_{s 2}\left(\frac{S k+s}{S K}\right)^{2} \\
& +\alpha_{s 3}\left(\frac{S k+s}{S K}\right)^{3}+R_{3, S k+s}
\end{aligned}
$$

Under $\mathrm{H}_{0}, \boldsymbol{\alpha}_{s}=\mathbf{0}$, which is the new null hypothesis. In order to define the test statistic we first extend the score defined in Lemma 3 by the vector (23). Assuming $R_{3, S k+s}=0$ for the same reason as before, the corresponding information matrix is defined in the following result:

Lemma 12 The information matrix of the auxiliary log-likelihood of the SSM-AR model with two transitions in which the coefficient of the sth seasonal dummy variable in the second transition is defined by (30), equals

$$
\begin{aligned}
\mathbf{J}^{A}\left(\boldsymbol{\alpha}_{s}^{0}, \boldsymbol{\theta}_{s}^{0}, \boldsymbol{\phi}^{0}\right) & =\mathrm{E} \lim _{K \rightarrow \infty} \mathbf{s}_{S K}\left(\boldsymbol{\alpha}_{s}, \boldsymbol{\theta}_{s}^{0}, \boldsymbol{\phi}^{0}\right) \mathbf{s}_{S K}^{\prime}\left(\boldsymbol{\alpha}_{s}, \boldsymbol{\theta}_{s}^{0}, \boldsymbol{\phi}^{0}\right) \\
& =\left[\begin{array}{ccc}
\mathbf{J}^{A}\left(\boldsymbol{\alpha}_{s}\right) & \mathbf{J}^{A}\left(\boldsymbol{\alpha}_{s}, \boldsymbol{\theta}_{s}^{0}\right) & \mathbf{J}^{A}\left(\boldsymbol{\alpha}_{s}, \boldsymbol{\phi}^{0}\right) \\
& \mathbf{J}^{M}\left(\boldsymbol{\theta}_{s}^{0}\right) & \mathbf{J}^{M}\left(\boldsymbol{\theta}_{s}^{0}, \boldsymbol{\phi}^{0}\right) \\
& \mathbf{J}^{M}\left(\boldsymbol{\phi}^{0}\right)
\end{array}\right]
\end{aligned}
$$

where $\mathbf{J}^{M}\left(\boldsymbol{\theta}_{s}^{0}\right), \mathbf{J}^{M}\left(\boldsymbol{\theta}_{s}^{0}, \boldsymbol{\phi}^{0}\right)$ and $\mathbf{J}^{M}\left(\boldsymbol{\phi}^{0}\right)$ are defined as in (16), (17) and (18), respectively, with $\sigma^{2}\left(\frac{S k+s}{S K}\right)=\sigma^{2}, \mathbf{J}^{A}\left(\boldsymbol{\alpha}_{s}\right)$ is defined as in (24) and $\mathbf{J}^{A}\left(\boldsymbol{\alpha}_{s}, \boldsymbol{\phi}^{0}\right)$ as in (25), and

$$
\mathbf{J}^{A}\left(\boldsymbol{\alpha}_{s}, \boldsymbol{\theta}_{s}^{0}\right)=\lim _{K \rightarrow \infty} \mathrm{Es}_{K}\left(\boldsymbol{\alpha}_{s}\right) \mathbf{s}_{K}^{\prime}\left(\boldsymbol{\theta}_{s}\right)=\int_{0}^{1} \mathbf{r} \frac{\partial \delta_{s}(r)}{\partial \boldsymbol{\theta}_{s}^{\prime}} d r .
$$

Proof. See Appendix A.

Let $\mathbf{J}\left(\boldsymbol{\alpha}_{s}, \boldsymbol{\theta}_{s}^{0}, \boldsymbol{\phi}^{0}\right)=\left[\mathbf{J}\left(\boldsymbol{\alpha}_{s}, \boldsymbol{\theta}_{s}^{0}\right), \mathbf{J}\left(\boldsymbol{\alpha}_{s}, \boldsymbol{\phi}^{0}\right)\right]$ and

$$
\mathbf{J}_{s}^{M}=\left[\begin{array}{cc}
\mathbf{J}^{M}\left(\boldsymbol{\theta}_{s}^{0}\right) & \mathbf{J}^{M}\left(\boldsymbol{\theta}_{s}^{0}, \boldsymbol{\phi}^{0}\right) \\
& \mathbf{J}^{M}\left(\boldsymbol{\phi}^{0}\right)
\end{array}\right] .
$$
form:

Using this notation, the resulting test statistic $L M_{s 2}$ has the following

$$
\begin{aligned}
L M_{s 2}= & K\left\{\frac{1}{\sigma^{2} K} \sum_{k=0}^{K-1} \widehat{\varepsilon}_{S k+s} \mathbf{k}_{S k+s}^{\prime}\right\}\left\{\mathbf{J}^{A}\left(\boldsymbol{\alpha}_{s}\right)-\mathbf{J}^{A}\left(\boldsymbol{\alpha}_{s}, \boldsymbol{\theta}_{s}^{0}, \boldsymbol{\phi}^{0}\right)\left(\mathbf{J}_{s}^{M}\right)^{-1}\right. \\
& \left.\times \mathbf{J}^{A}\left(\boldsymbol{\phi}^{0}, \boldsymbol{\theta}_{s}^{0}, \boldsymbol{\alpha}_{s}\right)\right\}^{-1}\left\{\frac{1}{\sigma^{2} K} \sum_{k=0}^{K-1} \widehat{\varepsilon}_{S k+s} \mathbf{k}_{S k+s}\right\}
\end{aligned}
$$


The statistic (33) has an asymptotic $\chi^{2}$-distribution with three degrees of freedom when $\mathrm{H}_{0}$ holds. It is conveniently calculated in the $T R^{2}$ form. As previously, (33) is made operational by replacing the blocks of the information matrix by their consistent estimators. It functions as a misspecification test (as does the constancy test) in model evaluation and should be used in all applications of the SSM-AR model. As such, it is more useful than a joint test against one more transition in all seasonal coefficients. The latter test is therefore not discussed here.

The assumption of independent errors is tested by the Godfrey-Breusch test of no error autocorrelation adapted to the situation in which the estimated model is an SSM-AR model with constant error variance. Since the errors are likely to contain heteroskedasticity, the test statistic is robustified in the same way as the aforementioned tests.

\subsection{Testing constancy of unit error variances}

Once the mean has been specified and estimated assuming that the error variance is not time-varying, it is time to specify the structure of the variance. The error variance of the SSM-AR model is defined in Section 2 by (6), (7) and (8). As the number of transitions in (7) is not known a priori, it has to be determined from the data. This number may be zero, which is why the first step consists of testing the hypothesis that $\sigma_{s}^{2}\left(\frac{S k+s}{S K}\right)=\sigma_{s 0}^{2}$ for each unit $s$ separately. Separate testing is motivated except for practical reasons also by the fact that due to orthogonality of the seasonal error variances the statistic for testing the joint hypothesis of all seasonal error variances being constant would be the sum of the individual statistics. As in the case of the time-varying intercept, the testing problem is nonstandard. It has been already discussed in Silvennoinen and Teräsvirta (2016). Their solution is to circumvent the identification problem by approximating the transition function (8) by a Taylor expansion around the null hypothesis $\gamma_{s 1}^{(v)}=0$. The same solution is applied here, see the Appendix for details. If the Taylor expansion is of order $m$, the resulting test statistic has an asymptotic $\chi^{2}$ distribution with $m$ degrees of freedom when the null hypothesis is true.

Analogously to the specification of the mean it would be possible and rather straightforward to extend the test to the situation in which a unit error variance with one transition is tested against two. This possibility is, however, not discussed here. 


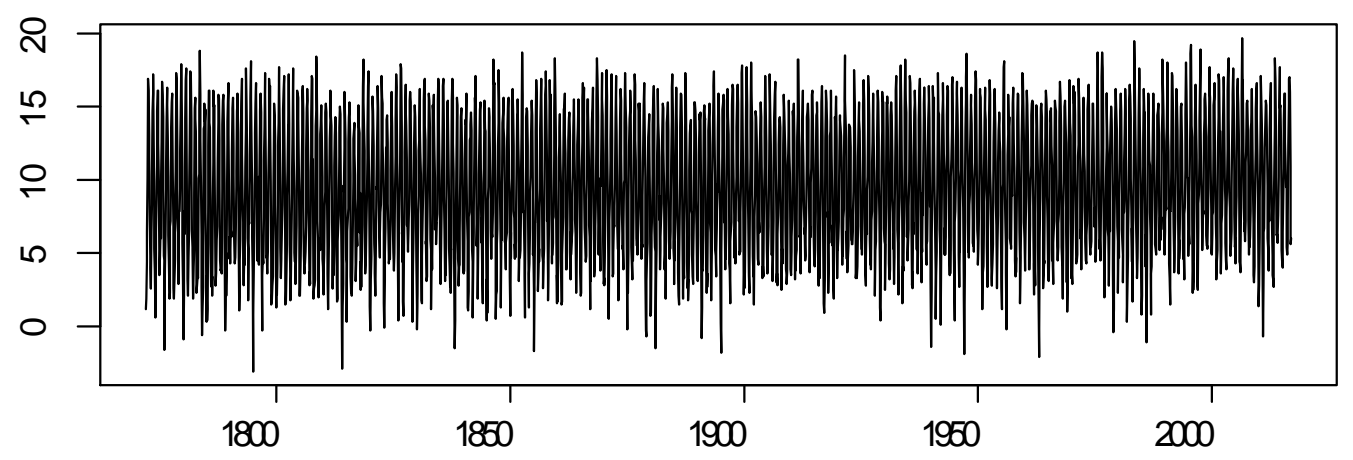

Figure 1: Monthly average temperatures in central England, 1772-2016

\section{Modelling the Central England tempera- ture series}

\subsection{The series}

The central England temperature series is one of the longest existing monthly temperature series. Because of its length and frequency it offers a possibility to study effects of climate change on temperatures by season. It is updated and available at http://www . metoffice.gov.uk/hadobs/hadcet/data/download.html. As mentioned in the Introduction, the series has for varying subperiods been analysed by several researchers. Following Proietti and Hillebrand (2017), we disregard the earliest observations because of quality issues and study the series beginning in 1772. The observations for the years 1772-2016 are plotted in Figure 1. The individual monthly averages are graphed in Figure 2. The graph shows variation in the monthly range of temperatures. There is more variation in the winter than in the summer. Here the interest lies in considering systematic variation in the errors.

\subsection{Testing constancy of seasonal mean temperatures}

Building an SSM-AR model begins for the CET series begins by determining the lag structure of the model. In a model with constant coefficient dummy variables BIC selects two lags, and this lag length is subsequently retained. Next, the null hypothesis of joint constancy of the coefficients of dummy variables is strongly rejected. The test results for individual months based on the robust test, see Section 6.3, appear in Table 1. Constancy is rejected at the $5 \%$ significance level for eight months for all three polynomial orders, 


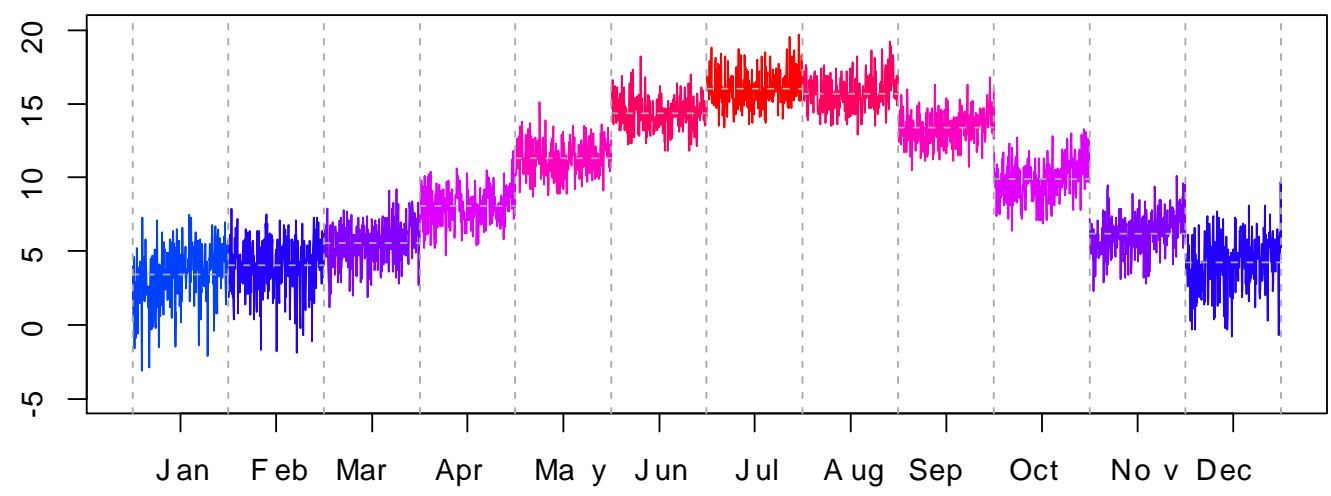

Figure 2: Temperatures of the CET series month by month, 1772-2016

the only exception being the third-order expansion based test for December. Constancy is not rejected for February, April, May and June, whereas September, October, November, January and March yield the strongest rejections. The high $p$-value for February may appear strange and will be returned to later. The conclusion from the tests is that a time-varying coefficient is introduced for eight dummies, February and the three months from April till June excluded. As discussed in Section 6.3, these coefficients are now estimated with one transition in each.

Interestingly, the rejections at the $5 \%$ level of significance in the tests coincide with certain test results reported in Proietti and Hillebrand (2017). The authors test stationarity of monthly temperatures against the alternative that the temperature has a stochastic trend component using the $5 \%$ level. They use the test by Nyblom and Mäkeläinen (1983) and reject the null hypothesis for the same eight months as does our stability test. The SSM-AR model with seasonal dummy variables is nonstationary but stable if its parameters are constant over time. Rejecting constancy in favour of deterministic shifts or 'trends' seems to bear a clear relationship to rejecting stationarity against a stochastic trend in the monthly model used by Proietti and Hillebrand (2017).

\subsection{Estimating the seasonal shifting mean autoregres- sive model}

Estimation of the mean begins by obtaining initial values using a two-dimensional grid (assuming the transition function (3)) for each month, as discussed in Section 5. The autoregressive terms contribute rather little to the explana- 


\begin{tabular}{rrrr}
\hline & 1 & 2 & 3 \\
\hline Jan & 0.0001 & 0.0006 & 0.0018 \\
Feb & 0.3969 & 0.5469 & 0.4568 \\
Mar & 0.0010 & 0.0003 & 0.0008 \\
Apr & 0.6206 & 0.2929 & 0.4364 \\
May & 0.9197 & 0.2274 & 0.3771 \\
Jun & 0.2023 & 0.2047 & 0.3564 \\
Jul & 0.0350 & 0.0080 & 0.0178 \\
Aug & 0.0407 & 0.0011 & 0.0024 \\
Sep & 0.0028 & 0.0024 & 0.0061 \\
Oct & 0.0000 & 0.0000 & 0.0001 \\
Nov & 0.0000 & 0.0001 & 0.0002 \\
Dec & 0.0102 & 0.0254 & 0.0584 \\
\hline
\end{tabular}

Table 1: $p$-values of monthly constancy tests for the Central England temperature series, 1772-2016

tion and even when their parameters slightly change from one grid estimation to the next, this does not pose problems.

Nonlinear estimation is carried out using our $\mathrm{R}$ code. The estimated equations can be found in Appendix C. In estimation, we set an upper bound for the slope parameter $\gamma$ to equal 40. This reflects our belief that long-run shifts in temperature are not completely abrupt and helps numerically in certain situations in which there is joint uncertainty about the speed and location of the change. In a couple of cases the transition is very smooth, which causes numerical problems in estimating the location parameter $c$ of the transition function. Thus, when the estimate of $c$ tends to exceed one, it is artificially restricted to this value. This reduces estimation uncertainty for both $\delta_{1}$, the coefficient of the transition function, and the location parameter $\gamma$.

The estimated model after the first iteration of the zig-zag algorithm can be found in Table 7 . Since they change only minimally after the first iteration, other than first iteration estimates are not reported. The main interest lies in the estimated monthly average temperatures which appear in Figure 3. Warming may be roughly divided in two categories. One is the 19 th century warming of winters that is visible in November, December and January. For December and January the location parameter is estimated to lie within the period 1820-1850. For March and the months from July to October the main part of warming occurs in the 20th century. The shape of the average for November suggests that this month's averages are a mixture of the 19th and the 20th century warming. This agrees with the fact that 

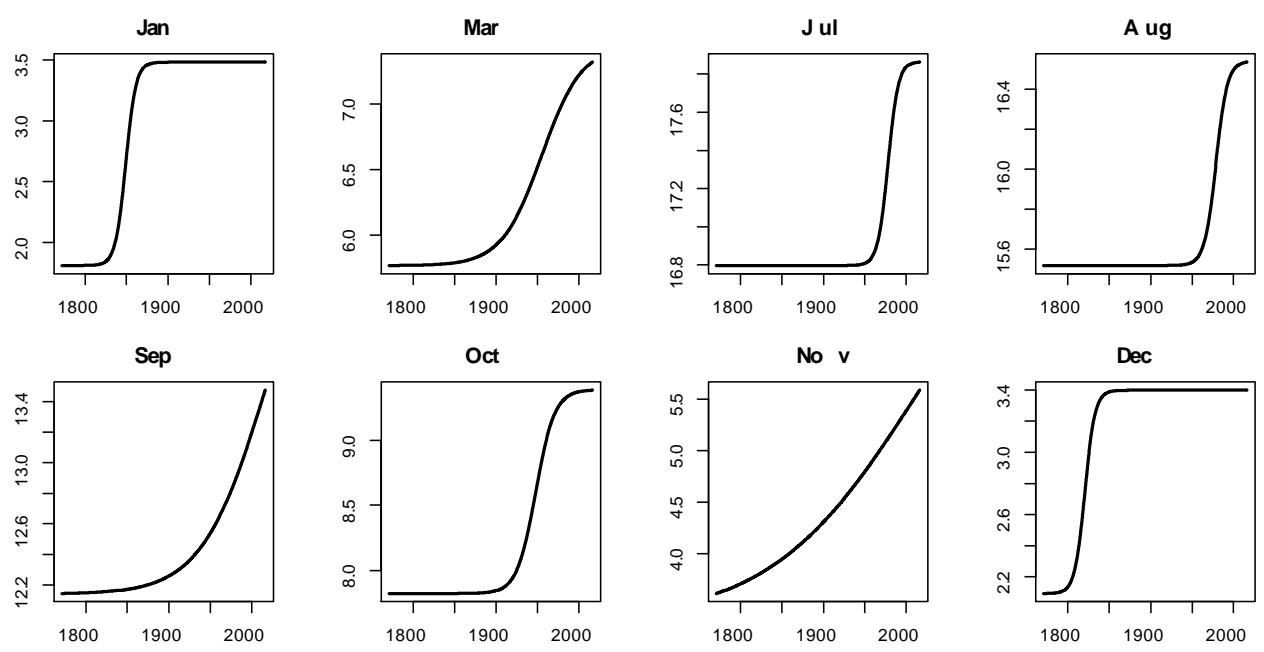

Figure 3: Estimated monthly Central England mean temperatures for the eight months in which the mean is time-varying, 1772-2016

the averages for the preceding months from July to October are dominated by the 20th century warming whereas the December and January warming occurs in the 19th century. This early winter warming coincides with the end of the so-called Little Ice Age around the mid-nineteenth century, so there may be a connection, but this is of course far from certain.

Changes in mean temperatures, measured in centigrade, for the whole period as well as for subperiods 1772-1899 and 1900-2016 can be found in Table 2. It is seen that July and August have warmed up later and less than September and October, and March for that matter. The values for March and September look quite similar. The 'no change' result for February may be explained by the disappearance of the 19th century winter effect, while 20th century warming has not yet appeared as it has in March. Why the tendency visible in March is not there for April, May and June is not clear. Warming in these three months has at any rate been too weak to be detected by our statistical model.

Before drawing more definite conclusions we have to perform another specification test, that is, we have to check whether changes in monthly averages are adequately described by a model with a single transition. For this purpose we test one transition against two as discussed in Section 6.4. The results can be found in Table 3. They suggest that the model with a single transition for the eight months with time-varying seasonal coefficients is adequate in the sense that no more transitions are required to describe the 


\begin{tabular}{rrrr}
\hline Month & $1772-2016$ & $1772-1899$ & $1900-2016$ \\
\hline Jan & 1.6682 & 1.6679 & 0.0004 \\
Feb & 0.0000 & 0.0000 & 0.0000 \\
Mar & 1.5551 & 0.1529 & 1.4021 \\
Apr & 0.0000 & 0.0000 & 0.0000 \\
May & 0.0000 & 0.0000 & 0.0000 \\
Jun & 0.0000 & 0.0000 & 0.0000 \\
Jul & 1.0670 & 0.0000 & 1.0670 \\
Aug & 1.0178 & 0.0000 & 1.0178 \\
Sep & 1.3315 & 0.1145 & 1.2170 \\
Oct & 1.5661 & 0.0214 & 1.5447 \\
Nov & 1.9737 & 0.6904 & 1.2833 \\
Dec & 1.3079 & 1.3079 & 0.0000 \\
\hline
\end{tabular}

Table 2: Estimated monthly mean temperature change for the years 17722016 and subperiods 1772-1899 and 1900-2016

behaviour of the coefficients. This conclusion does not depend on the order of the Taylor expansion in the test statistic (33).

Adequacy of the mean equation is also checked by testing the null hypothesis of no error autocorrelation. The results in Table 4 are based on the robust version Godfrey-Breusch test of no error autocorrelation modified for residuals from the SSM-AR model. It appears that the errors are not autocorrelated. This test a well as the test of one transition against two suggest that the mean part of the model is correctly specified.

\subsection{Specifying and estimating time-varying error vari- ance}

After estimation of the mean the next step is to model the error variance. Its monthly marginal distributions are plotted in Figure 4. Visual inspection suggests that there is more unexplained variation in winter months than in summer ones. The errors are clearly heteroskedastic, which the definition (6) of the error variance takes into account. The question to be investigated is whether the error variance $\sigma_{S k+s}^{2}$ considered for each month $s$ separately varies over time. This is studied by assuming that if it does, the change can be characterised by the model (6). Results from the test discussed in Section 6.5 appear in Table ?? and show that at the 5\% level, constancy is rejected only for April and May. Next, the effect of outliers on these results is studied as discussed in Appendix B. The errors deviating from zero by more than 


\begin{tabular}{lrrr}
\hline & 1 & 2 & 3 \\
\hline Jan & 0.6582 & 0.3729 & 0.2887 \\
Mar & 0.8790 & 0.9391 & 0.9834 \\
Jul & 0.8823 & 0.7706 & 0.3389 \\
Aug & 0.7637 & 0.3950 & 0.1167 \\
Sep & 0.9104 & 0.9262 & 0.9403 \\
Oct & 0.8611 & 0.8899 & 0.7974 \\
Nov & 0.9814 & 0.9940 & 0.8633 \\
Dec & 0.5810 & 0.4561 & 0.6193 \\
\hline
\end{tabular}

Table 3: $p$-values of the $F$ test of one transition against two month by month, using the first, second and third order Taylor expansion to approximate the second transition

\begin{tabular}{lr}
\hline Maximum lag & p-value \\
\hline 1 & 0.9188 \\
2 & 0.8610 \\
3 & 0.9002 \\
6 & 0.2300 \\
12 & 0.2281 \\
\hline
\end{tabular}

Table 4: $p$-values of the test of no error autocorrelation of selected orders up to 12

\pm 3 standard deviations are labelled as outliers and the tests repeated. The only change in results is that the $p$-values increase weakening the evidence of nonconstancy of the error variance for May. (April does not have any outliers, May has one, see Appendix D, Table 8). The first-order Taylor expansion test still yields a $p$-value slightly below 0.05 , whereas the other two values are above this level. Interestingly, the lowest $p$-values fall on months for which stability of the coefficient of seasonal dummy variable is not rejected.

Estimated error variances appear in Table 6. It is seen that the transitions are very abrupt, and in both cases the variance decreases after the shift or break. The break dates, the year 1814 for April and 1923 for May, do not seem to have a clear interpretation. In general, the estimated variances agree with Figure 4.

\section{Final remarks}

In this work we develop a flexible nonlinear model capable of describing changes in the seasonal pattern of a time series over time. It is applied to the 


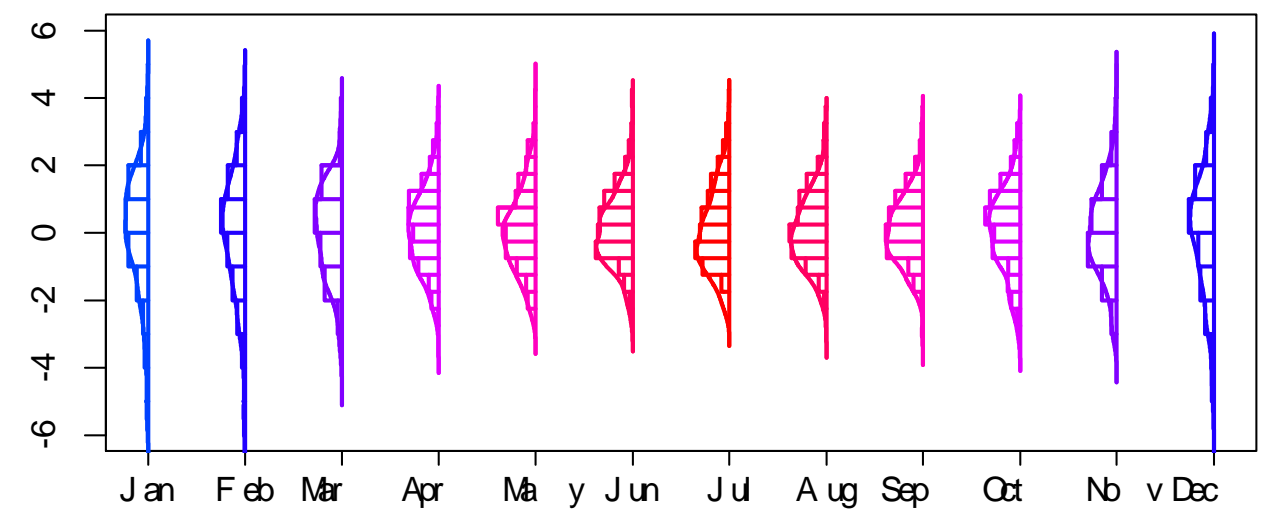

Figure 4: Histograms of residuals of the estimated SSM-AR model with constant error variance by month, 1772-2016

CET series using the same time period as Proietti and Hillebrand (2017) did but extended to the end of 2016. The main result is that with one exception, temperatures for the months from July to March have increased and that the warming has been strongest in the winter months. The results also show that there are differences in the timing of the increases in that temperatures in November, December and January have risen earlier than the rest. The end of the Little Ice Age may have played a role there, but we cannot be sure of that. Nor do we know why February temperatures seem not to have gone up.

Comparing these results with ones from other studies is not straightforward. Harvey and Mills (2003) use a different time periods beginning 1723 and ending 1999 and quarterly series. However, if we exclude the early years and consider their graphs estimated using local polynomials from 1772 onwards, similarities arise. Winter months (December, January, February) have been warming up already in the 19th century, whereas the others have not. Warming has been weak in the summer (June, July, August), whereas the late surge in the spring (March, April, May) is not visible in our results.

The monthly series of Vogelsang and Franses (2005) begins more than 100 years earlier than ours. There is some overlap in conclusions, see their Table 8, but also disagreement. Perhaps the most conspicuous similarity between our results and the ones in Proietti and Hillebrand (2017) is that in their structural time series model the deterministic drift component is strongest in November, December and January, i.e., in months for which we found early (19th century) warming. Since the structure of their model is rather different from ours, comparisons between results are not easy to make. It 


\begin{tabular}{rrrr}
\hline & 1 & 2 & 3 \\
\hline Jan & 0.2274 & 0.4706 & 0.5328 \\
Feb & 0.8532 & 0.4817 & 0.6219 \\
Mar & 0.2604 & 0.3469 & 0.2236 \\
Apr & 0.0831 & 0.0302 & 0.0703 \\
May & 0.0322 & 0.0902 & 0.1340 \\
Jun & 0.1183 & 0.2745 & 0.4152 \\
Jul & 0.9711 & 0.9792 & 0.9299 \\
Aug & 0.7816 & 0.9410 & 0.9673 \\
Sep & 0.4994 & 0.6004 & 0.7963 \\
Oct & 0.5983 & 0.8135 & 0.7642 \\
Nov & 0.7158 & 0.5079 & 0.3725 \\
Dec & 0.8028 & 0.8245 & 0.2504 \\
\hline
\end{tabular}

Table 5: $p$-values of tests of constancy of error variance by month based on the first, second and third order Taylor expansion

may be repeated, however, that outcomes of stationarity tests in their work are quite comparable to results of stability tests in this paper.

This analysis concentrates on the monthly CET time series, and the results from our model can hardly be generalised. Local conditions in the area have probably had a considerable effect on the outcomes. Hillebrand and Proietti (2017) are already considering 16 Northern Hemispheric series, and it would be interesting to do something similar in the present framework. This would involve considering a multivariate SSM-AR model, which could among other things make it possible to study correlation dynamics between different series. Extensions of this kind will be left for further work.

\section{References}

Ajmi, A. N., Ben Nasr, A. and Boutahar, M.: 2008, Seasonal nonlinear long memory model for the US inflation rates, Computational Economics 31, 243-254.

Amado, C. and Teräsvirta, T.: 2013, Modelling volatility by variance decomposition, Journal of Econometrics 175, 153-165.

Baek, Y. I., Cho, J. S. and Phillips, P. C. B.: 2015, Testing linearity using power transforms of regressors, Journal of Econometrics 187, 376-384. 


\begin{tabular}{lrrr}
\hline & Estimate & Std. Error & t value \\
\hline$\sigma_{1,0}^{2}$ & 3.19 & 0.290 & 11.0 \\
$\sigma_{2,0}^{2}$ & 3.13 & 0.285 & 11.0 \\
$\sigma_{3,0}^{2}$ & 1.80 & 0.162 & 11.1 \\
$\sigma_{4,0}^{2}$ & 2.05 & 0.408 & 5.02 \\
$\omega_{4}$ & -0.809 & 0.428 & -1.89 \\
$\gamma_{4}$ & 671 & 1710 & \\
$c_{4}$ & 0.175 & 0.007 & \\
$\sigma_{5,0}^{2}$ & 1.60 & 0.187 & 8.56 \\
$\omega_{5}$ & -0.606 & 0.244 & -2.48 \\
$\gamma_{5}$ & 44.0 & 97.3 & \\
$c_{5}$ & 0.616 & 0.040 & \\
$\sigma_{6,0}^{2}$ & 1.08 & 0.097 & 11.1 \\
$\sigma_{7,0}^{2}$ & 1.31 & 0.119 & 11.0 \\
$\sigma_{8,0}^{2}$ & 1.02 & 0.092 & 11.1 \\
$\sigma_{9,0}^{2}$ & 1.01 & 0.090 & 11.1 \\
$\sigma_{10,0}^{2}$ & 1.49 & 0.134 & 11.1 \\
$\sigma_{11,0}^{2}$ & 1.78 & 0.159 & 11.2 \\
$\sigma_{12,0}^{2}$ & 3.13 & 0.284 & 11.0 \\
\hline
\end{tabular}

No $t$-values are reported for the estimates of $\gamma$ and $c$. For $\widehat{c}$, significant deviations from zero are of no interest. As to $\widehat{\gamma}$, due to the identification problem the asymptotic distribution of the $t$-statistic is unknown, and reporting a $t$-value is therefore misleading.

Table 6: Estimates of the variance component of the SSM-AR model for the monthly CET series, 1772-2016

Craig, L. A. and Holt, M. T.: 2008, Mechanical refrigeration, seasonality, and the hog-corn cycle in the United States: 1870-1940, Explorations in Economic History 45, 30-50.

Craig, L. A. and Holt, M. T.: 2017, The impact of mechanical refrigeration on market integration: The U.S. egg market, 1890-1911, Explorations in Economic History 66, 85-105.

Davies, R. B.: 1977, Hypothesis testing when a nuisance parameter is present only under the alternative, Biometrika 64, 247-254.

Franses, P. H. and de Bruin, P.: 2000, Seasonal adjustment and the business cycle in unemployment, Studies in Nonlinear Dynamics $\&$ Econometrics 4, Iss. 2, Article 2. 
González, A. and Teräsvirta, T.: 2008, Modelling autoregressive processes with a shifting mean, Studies in Nonlinear Dynamics and Econometrics 12, Iss. 1, Article 1.

Hansen, B. E.: 1996, Inference when a nuisance parameter is not identified under the null hypothesis, Econometrica 64, 413-430.

Harvey, D. I. and Mills, T. C.: 2003, Modelling trends in central England temperatures, Journal of Forecasting 22, 35-47.

Hillebrand, E. and Proietti, T.: 2017, Phase changes and seasonal warming in early instrumental temperature records, Journal of Climate 30, 67956821.

Luukkonen, R., Saikkonen, P. and Teräsvirta, T.: 1988, Testing linearity against smooth transition autoregressive models, Biometrika 75, 491499.

Manley, G.: 1974, Central England temperatures: monthly means 1659 to 1973, Quarterly Journal of the Royal Meteorological Society 100, 389405.

Newey, W. K. and McFadden, D. L.: 1994, Large sample estimation and hypothesis testing, in R. F. Engle and D. L. McFadden (eds), Handbook of Econometrics, Vol. 4, Elsevier Science, Amsterdam, pp. 2111-2245.

Nyblom, J. and Mäkeläinen, T.: 1983, Comparisons of tests for the presence of random walk coefficients in a simple linear model, Journal of the American Statistical Association 78, 856-864.

Oberhofer, W. and Kmenta, J.: 1974, A general procedure for obtaining maximum likelihood estimates in generalized regression models, Econometrica 42, 579-590.

Parker, D. E., Legg, T. P. and Folland, C. K.: 1992, A new daily central England temperature series,1772-1991, International Journal of Climatology 12, 317-342.

Proietti, T. and Hillebrand, E.: 2017, Seasonal changes in central England temperatures, Journal of the Royal Statistical Society, A 180, 769-791.

Sargan, J. D.: 1964, Wages and prices in the United Kingdom: A study of econometric methodology, in P. E. Hart, G. Mills and J. K. Whitaker (eds), Econometric Analysis for National Economic Planning, Butterworth, London, pp. 25-54. 
Silvennoinen, A. and Teräsvirta, T.: 2016, Testing constancy of unconditional variance in volatility models by misspecification and specification tests, Studies in Nonlinear Dynamics and Econometrics 20, 347-364.

Teräsvirta, T., Tjøstheim, D. and Granger, C. W. J.: 2010, Modelling Nonlinear Economic Time Series, Oxford University Press, Oxford.

van Dijk, D., Franses, P. H. and Lucas, A.: 1999, Testing for ARCH in the presence of additive outliers, Journal of Applied Econometrics 14, 539562.

van Dijk, D., Strikholm, B. and Teräsvirta, T.: 2003, The effects of institutional and technological change and business cycle fluctuations on seasonal patterns in quarterly industrial production series, Econometrics Journal 6, 79-98.

Vogelsang, T. J. and Franses, P. H.: 2005, Are winters getting warmer?, Environmental Modelling \& Software 20, 1449-1455.

Wooldridge, J. M.: 1990, A unified approach to robust, regression-based specification tests, Econometric Theory 6, 17-43.

\section{A Proofs}

Proof of Lemma 5. Consider

$$
\mathbf{J}_{K}^{M}\left(\boldsymbol{\theta}_{s}\right)=\mathrm{E} s_{K}\left(\boldsymbol{\theta}_{s}\right) s_{K}^{\prime}\left(\boldsymbol{\theta}_{s}\right)=\frac{1}{K} \sum_{k=0}^{K-1} \frac{\mathrm{E} \varepsilon_{S k+s}^{2}}{\sigma_{s}^{4}\left(\frac{S k+s}{S K}\right)} \frac{\partial \delta_{s}\left(\frac{S k+s}{S K}\right)}{\partial \boldsymbol{\theta}_{s}} \frac{\partial \delta_{s}\left(\frac{S k+s}{S K}\right)}{\partial \boldsymbol{\theta}_{s}^{\prime}}
$$

for $s=1, \ldots, S$. Let $k=[r K], 0<r \leq 1$. As $\mathrm{E} \varepsilon_{S k+s}^{2} / \sigma_{s}^{2}\left(\frac{S k+s}{S K}\right)=1$, the limit of (34) becomes

$$
\begin{aligned}
\mathbf{J}_{K}^{M}\left(\boldsymbol{\theta}_{s}\right) & =\sum_{k=0}^{K-1} \int_{\frac{S k+s}{S K}}^{\frac{S(k+1)+s}{S K}} \frac{1}{\sigma_{s}^{2}\left(\frac{S[r K]+s}{S K}\right)} \frac{\partial \delta_{s}\left(\frac{S[r K]+s}{S K}\right)}{\partial \boldsymbol{\theta}_{s}} \frac{\partial \delta_{s}\left(\frac{S[r K]+s}{S K}\right)}{\partial \boldsymbol{\theta}_{s}^{\prime}} \mathrm{d} r \\
& =\int_{\frac{s}{S K}}^{\frac{S K+s}{S K}} \frac{1}{\sigma_{s}^{2}\left(\frac{S[r K]+s}{S K}\right)} \frac{\partial \delta_{s}\left(\frac{S[r K]+s}{S K}\right)}{\partial \boldsymbol{\theta}_{s}} \frac{\partial \delta_{s}\left(\frac{S[r K]+s}{S K}\right)}{\partial \boldsymbol{\theta}_{s}^{\prime}} \mathrm{d} r \\
& \rightarrow \int_{0}^{1} \frac{1}{\sigma_{s}^{2}(r)} \frac{\partial \delta_{s}(r)}{\partial \boldsymbol{\theta}_{s}} \frac{\partial \delta_{s}(r)}{\partial \boldsymbol{\theta}_{s}^{\prime}} \mathrm{d} r=\mathbf{J}^{M}\left(\boldsymbol{\theta}_{s}\right) .
\end{aligned}
$$

as $K \rightarrow \infty$. 
Next we have

$$
\mathbf{J}_{S K}^{M}(\boldsymbol{\phi})=\mathrm{E}_{s_{S K}}(\boldsymbol{\phi}) s_{S K}^{\prime}(\boldsymbol{\phi})=\sum_{k=0}^{K-1} \sum_{s=1}^{S} \frac{1}{\sigma_{s}^{2}\left(\frac{S k+s}{S K}\right)} \mathrm{E}_{\mathbf{y}_{S k+s-1}} \mathbf{y}_{S k+s-1}^{\prime} .
$$

To consider the limit of (35), assuming A5 holds the $\ell$ th element of $y_{S k+s-m}$ can be written as

$$
\begin{aligned}
y_{S k+s-\ell} & =\phi^{-1}(\mathrm{~L}) \delta_{s-\ell}\left(\frac{S k+s-\ell}{S K}\right) D_{S k+s-\ell}^{(s-\ell)}+\phi^{-1}(\mathrm{~L}) \varepsilon_{S k+s-\ell} \\
& =\sum_{i=0}^{\infty} \psi_{i}\left\{\delta_{s-\ell}\left(\frac{S k+s-\ell-i}{S K}\right) D_{S k+s-\ell-i}^{(s-\ell)}+\varepsilon_{S k+s-\ell-i}\right\} \\
& =\sum_{i=0}^{\infty} \psi_{i S} \delta_{s-\ell}\left(\frac{S(k-i)+s-\ell}{S K}\right) D_{S(k-i)+s-\ell}^{(s-\ell)}+\sum_{i=0}^{\infty} \psi_{i} \varepsilon_{S k+s-\ell-i}
\end{aligned}
$$

for $\ell=m, n$, where $\phi^{-1}(\mathrm{~L})=\sum_{i=0}^{\infty} \psi_{i} \mathrm{~L}^{i}$ with $\psi_{0}=1$ and $\delta_{s-\ell}(x)=0$ for $x<0$. Now, $D_{S(k-i)+s-l}^{(s-\ell)}=1$ for $i=0,1, \ldots k$, and when $i=k$, then $s-\ell>0$. A necessary condition for $D_{S(k-i)+s-m}^{(s-m)} D_{S(k-j)+s-n}^{(s-n)}=1$ is $m=n$. More generally, $D_{S(k-i)+s-m}^{(s-m)} D_{S(k-j)+s-n}^{(s-n)}=1$ for $m=n$ and $i, j=0,1, \ldots, k$, where $k=0,1, \ldots, K$. Then

$$
y_{S k+s-m} y_{S k+s-n}=\sum_{i=0}^{\infty} \sum_{j=0}^{\infty} \psi_{i} \psi_{j} \varepsilon_{S k+s-m-i} \varepsilon_{S k+s-n-j}
$$

for $m \neq n$, and

$$
\begin{aligned}
y_{S k+s-m}^{2}= & \left\{\sum_{i=0}^{\infty} \psi_{i S} \delta_{s-m}\left(\frac{S(k-i)+s-m}{S K}\right)\right\}\left\{\sum_{j=0}^{\infty} \psi_{j S} \delta_{s-m}\left(\frac{S(k-j)+s-m}{S K}\right)\right\} \\
& +\sum_{i=0}^{\infty} \psi_{i} \varepsilon_{S k+s-m-i}\left(\sum_{j=0}^{\infty} \psi_{j} \varepsilon_{S k+s-m-j}\right) \\
= & \sum_{i=0}^{\infty} \sum_{j=0}^{\infty} \psi_{i S} \psi_{j S} \delta_{s-m}\left(\frac{S(k-i)+s-m}{S K}\right) \delta_{s-m}\left(\frac{S(k-j)+s-m}{S K}\right) \\
& \sum_{i=0}^{\infty} \sum_{j=0}^{\infty} \psi_{i} \psi_{j} \varepsilon_{S k+s-m-i} \varepsilon_{S k+s-m-j} .
\end{aligned}
$$


for $m=n$. Assuming $m-n=\nu \geq 0$,

$$
\begin{aligned}
\mathrm{E} y_{S k+s-n-\nu} y_{S k+s-n} & =\sum_{i=0}^{\infty} \sum_{j=0}^{\infty} \psi_{i} \psi_{j} \mathrm{E}_{S k+s-n-\nu-i} \varepsilon_{S k+s-n-j} \\
& =\sum_{i=0}^{\infty} \psi_{i} \psi_{i+\nu} \mathrm{E}_{S k+s-n-i}^{2} \\
& =\sum_{i=0}^{\infty} \psi_{i} \psi_{i+\nu} \sigma_{s-n}^{2}\left(\frac{S k+s-n-i}{S K}\right) D_{S k+s-n-i}^{(s-n)} \\
& =\sum_{i=0}^{\infty} \psi_{i S} \psi_{i S+\nu} \sigma_{s-n}^{2}\left(\frac{S(k-i)+s-n}{S K}\right) .
\end{aligned}
$$

Then

$$
\begin{aligned}
{\left[\mathbf{J}_{S K}^{M}(\phi)\right]_{n n}=} & \frac{1}{S K} \sum_{k=0}^{K-1} \sum_{s=1}^{S} \frac{1}{\sigma_{s}^{2}\left(\frac{S k+s}{S K}\right)} \mathrm{E} y_{S k+s}^{2} \\
= & \frac{1}{S K} \sum_{s=1}^{S} \sum_{k=0}^{K-1} \frac{1}{\sigma_{s}^{2}\left(\frac{S k+s}{S K}\right)} \sum_{i=0}^{\infty} \sum_{j=0}^{\infty} \psi_{i S} \psi_{j S} \\
& \times \delta_{s-n}\left(\frac{S(k-i)+s-n}{S K}\right) \delta_{s-n}\left(\frac{S(k-j)+s-n}{S K}\right) \\
& +\frac{1}{S K} \sum_{s=1}^{S} \sum_{k=0}^{K-1} \frac{1}{\sigma_{s}^{2}\left(\frac{S k+s}{S K}\right)} \sum_{i=0}^{\infty} \psi_{i S} \psi_{i S+\nu} \sigma_{s-n}^{2}\left(\frac{S(k-i)+s-n}{S K}\right) \\
= & \sum_{i=0}^{\infty} \sum_{j=0}^{\infty} \psi_{i S} \psi_{j S} \frac{1}{S K} \sum_{s=1}^{S} \sum_{k=0}^{K-1} \frac{1}{\sigma_{s}^{2}\left(\frac{S k+s}{S K}\right)} \\
& \times \delta_{s-n}\left(\frac{S(k-i)+s-n}{S K}\right) \delta_{s-n}\left(\frac{S(k-j)+s-n}{S K}\right) \\
& +\sum_{i=0}^{\infty} \psi_{i S} \psi_{i S+\nu} \frac{1}{S K} \sum_{k=0}^{K-1} \sum_{s=1}^{S} \frac{\sigma_{s-n}^{2}\left(\frac{S(k-1)+s-n}{S K}\right)}{\sigma_{s}^{2}\left(\frac{S k+s}{S K}\right)}
\end{aligned}
$$


Setting $k=[r K]$ in (36) yields

$$
\begin{aligned}
{\left[\mathbf{J}_{S K}^{M}(\boldsymbol{\phi})\right]_{n n}=} & \sum_{i=0}^{\infty} \sum_{j=0}^{\infty} \psi_{i S} \psi_{j S} \frac{1}{S K} \sum_{s=1}^{S} \sum_{k=0}^{K-1} \int_{\frac{S k+s}{S K}}^{\frac{S(k+1)}{S K}} \frac{1}{\sigma_{s}^{2}\left(\frac{S[r K]+s}{S K}\right)} \\
& \times \delta_{s-n}\left(\frac{S([r K]-i)+s-n}{S K}\right) \delta_{s-n}\left(\frac{S([r K]-j)+s-n}{S K}\right) \mathrm{d} r \\
& +\sum_{i=0}^{\infty} \psi_{i S} \psi_{i S+\nu} \frac{1}{S} \sum_{k=0}^{K-1} \sum_{s=1}^{S} \int_{\frac{S k+s}{S K}}^{\frac{S(k+1)+s}{S K}} \frac{\sigma_{s-n}^{2}\left(\frac{S([r K]-1)+s-n}{S K}\right)}{\sigma_{s}^{2}\left(\frac{S[r K]+s}{S K}\right)} \mathrm{d} r \\
= & \sum_{i=0}^{\infty} \sum_{j=0}^{\infty} \psi_{i S} \psi_{j S} \frac{1}{S} \sum_{s=1}^{S} \int_{\frac{s}{S K}}^{\frac{S K+s}{S K}} \frac{1}{\sigma_{s}^{2}\left(\frac{S[r K]+s}{S K}\right)} \\
& \times \delta_{s-n}\left(\frac{S([r K]-i)+s-n}{S K}\right) \delta_{s-n}\left(\frac{S([r K]-j)+s-n}{S K}\right) \mathrm{d} r \\
& ++\sum_{i=0}^{\infty} \psi_{i S} \psi_{i S+\nu} \frac{1}{S} \sum_{s=1}^{S} \int_{\frac{s}{S K}}^{\frac{S K+s}{S K}} \frac{\sigma_{s-n}^{2}\left(\frac{S([r K]-1)+s-n}{S K}\right)}{\sigma_{s}^{2}\left(\frac{S[r K]+s}{S K}\right)} \mathrm{d} r \\
\rightarrow & \sum_{i=0}^{\infty} \sum_{j=0}^{\infty} \psi_{i S} \psi_{j S+\nu} \frac{1}{S} \sum_{s=1}^{S} \int_{0}^{1} \frac{\delta_{s-n}^{2}(r)}{\sigma_{s}^{2}(r)} \mathrm{d} r+\sum_{i=0}^{\infty} \psi_{i S} \psi_{i S+\nu} .
\end{aligned}
$$

Finally,

$$
\begin{aligned}
\mathbf{J}_{S K}^{M}\left(\boldsymbol{\theta}_{s}, \boldsymbol{\phi}\right)= & \mathrm{E} s_{K}\left(\boldsymbol{\theta}_{s}\right) s_{S K}^{\prime}(\boldsymbol{\phi})=\frac{1}{K} \sum_{k=0}^{K-1} \frac{\mathrm{E} \varepsilon_{S k+s}^{2}}{\sigma_{s}^{4}\left(\frac{S k+s}{S K}\right)} \frac{\partial \delta_{s}\left(\frac{S k+s}{S K}\right)}{\partial \boldsymbol{\theta}_{s}} \mathrm{E}_{S k+s-1}^{\prime} \\
= & \frac{1}{\phi(1) K} \sum_{k=0}^{K-1} \frac{1}{\sigma_{s}^{2}\left(\frac{S k+s}{S K}\right)} \frac{\partial \delta_{s}\left(\frac{S k+s}{S K}\right)}{\partial \boldsymbol{\theta}_{s}}\left(\delta_{s-1}\left(\frac{S k+s-1}{S K}\right), \ldots,\right. \\
& \left.\delta_{s-p}\left(\frac{S k+s-p}{S K}\right)\right) \\
= & \frac{1}{\phi(1)} \sum_{k=0}^{K-1} \int_{\frac{S k+s}{S K}}^{\frac{S(k+1)+s}{S K}} \frac{1}{\sigma_{s}^{2}\left(\frac{S[r K]+s}{S K}\right)} \frac{\partial \delta_{s}\left(\frac{S[r K]+s}{S K}\right)}{\partial \boldsymbol{\theta}_{s}}\left(\delta_{s-1}\left(\frac{S[r K]+s-1}{S K}\right), \ldots,\right. \\
= & \frac{1}{\phi(1)} \int_{\frac{s}{S K}}^{\frac{S K+s}{S K}} \frac{1}{\sigma_{s}^{2}\left(\frac{S[r K]+s}{S K}\right)} \frac{\partial \delta_{s}\left(\frac{S[r K]+s}{S K}\right)}{\partial \boldsymbol{\theta}_{s}}\left(\delta_{s-1}\left(\frac{S[r K]+s-1}{S K}\right), \ldots,\right. \\
& \delta_{s-p}\left(\frac{S[r K]+s-p}{S K}\right) \mathrm{d} r \\
\rightarrow & \frac{1}{\phi(1)} \int_{0}^{1} \frac{1}{\sigma_{s}^{2}(r)} \frac{\partial \delta_{s}(r)}{\partial \boldsymbol{\theta}_{s}} \boldsymbol{\delta}_{s-1}^{\prime}(r) \mathrm{d} r=\mathbf{J}^{M}\left(\boldsymbol{\theta}_{s}, \boldsymbol{\phi}\right)
\end{aligned}
$$


as $K \rightarrow \infty$. This completes the proof.

Proof of Lemma 6. Consider

$$
\begin{aligned}
\mathbf{J}_{K}^{V}\left(\boldsymbol{\theta}_{s}^{(v) 0}\right) & =\mathrm{Es}_{K}\left(\boldsymbol{\theta}_{s}^{(v) 0}\right) \mathbf{s}_{K}\left(\boldsymbol{\theta}_{s}^{(v) 0}\right)^{\prime} \\
& =\frac{1}{4 K} \sum_{k=0}^{K-1} \mathrm{E}\left(\frac{\varepsilon_{S k+s}^{2}}{\sigma_{s}^{2}\left(\frac{S k+s}{S K}\right)}-1\right)^{2} \frac{1}{\sigma_{s}^{4}\left(\frac{S k+s}{S K}\right)} \frac{\partial \sigma^{2}\left(\frac{S k+s}{S K}\right)}{\partial \boldsymbol{\theta}_{s}^{(v)}} \frac{\partial \sigma^{2}\left(\frac{S k+s}{S K}\right)}{\partial \boldsymbol{\theta}_{s}^{(v) \prime}}
\end{aligned}
$$

for $s=1, \ldots, S$. As before, set $k=[r K], 0<r \leq 1$. Then the limit of (37) becomes

$$
\begin{aligned}
\mathbf{J}_{K}^{V}\left(\boldsymbol{\theta}_{s}^{(v) 0}\right) & =\frac{1}{2} \sum_{k=0}^{K-1} \int_{\frac{S k+s}{S K}}^{\frac{S(k+1)+s}{S K}} \frac{1}{\sigma_{s}^{4}\left(\frac{S[r K]+s}{S K}\right)} \frac{\partial \sigma^{2}\left(\frac{S[r K]+s}{S K}\right)}{\partial \boldsymbol{\theta}_{s}^{(v)}} \frac{\partial \sigma^{2}\left(\frac{S[r K]+s}{S K}\right)}{\partial \boldsymbol{\theta}_{s}^{(v) \prime}} \mathrm{d} r \\
& =\frac{1}{2} \int_{\frac{s}{S K}}^{\frac{S(k+1)+s}{S K}} \frac{1}{\sigma_{s}^{4}\left(\frac{S[r K]+s}{S K}\right)} \frac{\partial \sigma^{2}\left(\frac{S[r K]+s}{S K}\right)}{\partial \boldsymbol{\theta}_{s}^{(v)}} \frac{\partial \sigma^{2}\left(\frac{S[r K]+s}{S K}\right)}{\partial \boldsymbol{\theta}_{s}^{(v) \prime}} \mathrm{d} r \\
& \rightarrow \frac{1}{2} \int_{0}^{1} \frac{1}{\sigma_{s}^{4}(r)} \frac{\partial \sigma^{2}(r)}{\partial \boldsymbol{\theta}_{s}^{(v)}} \frac{\partial \sigma^{2}(r)}{\partial \boldsymbol{\theta}_{s}^{(v) \prime}} \mathrm{d} r=\mathbf{J}^{V}\left(\boldsymbol{\theta}_{s}^{(v) 0}\right)
\end{aligned}
$$

as $K \rightarrow \infty$.

Proof of Theorem 9. We prove this result by verifying the conditions of Theorem 2.5 in Newey and McFadden (1994, p. 2131):

Theorem 2.5 (Newey and McFadden). Suppose that $z_{S k+s}(k=0, \ldots, K-$ $1 ; s=1, \ldots, S)$ are iid with probability distribution function $f\left(z_{S k+s} \mid \boldsymbol{\theta}^{0}\right)$. If

(i) $\boldsymbol{\theta} \neq \boldsymbol{\theta}^{0}$, then $f\left(z_{S k+s} \mid \boldsymbol{\theta}^{0}\right) \neq f\left(z_{S k+s} \mid \boldsymbol{\theta}\right)$,

(ii) $\boldsymbol{\theta}^{0} \in \Theta$ which is compact, and the density is positive (bounded away from zero) for all $\boldsymbol{\theta} \in \Theta$,

(iii) $\ln f\left(z_{S k+s} \mid \boldsymbol{\theta}\right)$ is continuous at each $\boldsymbol{\theta} \in \Theta$ with probability one,

(iv) $\operatorname{Esup}_{\boldsymbol{\theta} \in \Theta}\left|\ln f\left(z_{S k+s} \mid \boldsymbol{\theta}\right)\right|<\infty$,

then the maximum likelihood estimator $\widehat{\boldsymbol{\theta}} \stackrel{p}{\rightarrow} \boldsymbol{\theta}^{0}$ as $K \rightarrow \infty$.

Assumption (i) is satisfied due to A1 and A2. Assumption (ii) is valid due to A3 and A4, and (iii) follows from A5 and Lemma 3. (iv) Applying the mean value theorem, the triangle inequality and the Cauchy-Schwarz inequality to $\left|\ln f\left(z_{S k+s} \mid \boldsymbol{\theta}\right)\right|$ yields

$$
\begin{aligned}
\left|\ln f\left(z_{S k+s} \mid \boldsymbol{\theta}\right)\right| & =\left|\ln f\left(z_{S k+s} \mid \boldsymbol{\theta}^{0}\right)+\frac{\partial}{\partial \boldsymbol{\theta}^{\prime}} \ln f\left(z_{S k+s} \mid \overline{\boldsymbol{\theta}}\right)\left(\boldsymbol{\theta}-\boldsymbol{\theta}^{0}\right)\right| \\
& \leq\left|\ln f\left(z_{S k+s} \mid \boldsymbol{\theta}^{0}\right)\right|+\left\|\frac{\partial}{\partial \boldsymbol{\theta}^{\prime}} \ln f\left(z_{S k+s} \mid \overline{\boldsymbol{\theta}}\right)\right\| \times\left\|\left(\boldsymbol{\theta}-\boldsymbol{\theta}^{0}\right)\right\| \\
& \leq C_{1}+C_{2} C_{3}<\infty
\end{aligned}
$$


where $\overline{\boldsymbol{\theta}}$ is an intermediate value between $\boldsymbol{\theta}$ and $\boldsymbol{\theta}^{0},\left|\ln f\left(z_{S k+s} \mid \boldsymbol{\theta}^{0}\right)\right| \leq C_{1}$ because of A4, and $\left\|\frac{\partial}{\partial \boldsymbol{\theta}^{\prime}} \ln f\left(z_{S k+s} \mid \overline{\boldsymbol{\theta}}\right)\right\| \leq C_{2}$ because of Lemma 3. Furthermore, $\left\|\boldsymbol{\theta}-\boldsymbol{\theta}^{0}\right\| \leq C_{3}$ because $\Theta$ is compact (A3), so the elements of $\left\|\boldsymbol{\theta}-\boldsymbol{\theta}^{0}\right\|$ are bounded for $\boldsymbol{\theta} \in \Theta$. In (38), $C_{i}, i=1,2,3$, are generic positive constants. As $\left|\ln f\left(z_{S k+s} \mid \boldsymbol{\theta}\right)\right|$ is finite for all $\boldsymbol{\theta} \in \Theta$, the expectation of its supremum is finite as well.

Proof of Theorem 10. We prove the theorem by verifying the conditions of Theorem 3.3 in Newey and McFadden (1994, p. 2146):

Theorem 3.3 (Newey and McFadden). Suppose that hypotheses of Theorem 2.5 are satisfied and

(i) $\boldsymbol{\theta}^{0}$ is an interior point of $\Theta$,

(ii) $f(z \mid \boldsymbol{\theta})$ is twice continuously differentiable and $f(z \mid \boldsymbol{\theta})>0$ in a neighbourhood $\mathcal{N}$ of $\boldsymbol{\theta}^{0}$,

(iii) $\int \sup _{\boldsymbol{\theta} \in \mathcal{N}}\left\|\frac{\partial}{\partial \boldsymbol{\theta}} f(z \mid \boldsymbol{\theta})\right\| \mathrm{d} z<\infty$ and $\int \sup _{\boldsymbol{\theta} \in \mathcal{N}}\left\|\frac{\partial^{2}}{\partial \boldsymbol{\theta} \partial \boldsymbol{\theta}^{\prime}} f(z \mid \boldsymbol{\theta})\right\| \mathrm{d} z<\infty$,

(iv) $\mathbf{J}\left(\boldsymbol{\theta}^{0}\right)=\mathrm{E}\left\{\ln \frac{\partial}{\partial \boldsymbol{\theta}} f\left(z \mid \boldsymbol{\theta}^{0}\right)\right\}\left\{\frac{\partial}{\partial \boldsymbol{\theta}^{\prime}} \ln f\left(z \mid \boldsymbol{\theta}^{0}\right)\right\}$ exists and is nonsingular,

(v) $\operatorname{Esup}_{\boldsymbol{\theta} \in \mathcal{N}}\left\|\frac{\partial^{2}}{\partial \boldsymbol{\theta} \partial \boldsymbol{\theta}^{\prime}} \ln f(z \mid \boldsymbol{\theta})\right\|<\infty$, where $\mathcal{N}$ is a neighbourhood of $\boldsymbol{\theta}^{0}$. Then $\sqrt{K}\left(\widehat{\boldsymbol{\theta}}-\boldsymbol{\theta}^{0}\right) \stackrel{d}{\rightarrow} N\left(0, \mathbf{J}^{-1}\left(\boldsymbol{\theta}^{0}\right)\right)$.

Condition (i) follows from A3 and (ii) from A4 and Lemma 7. Condition (iii) is a standard regularity condition and is satisfied. In order to verify (iv), we consider blocks of (15). First note that $\mathbf{J}_{K}\left(\boldsymbol{\theta}_{M}^{0}\right)=\mathrm{E} s_{S K}\left(\boldsymbol{\theta}_{M}^{0}\right) s_{S K}^{\prime}\left(\boldsymbol{\theta}_{M}^{0}\right)$ is nonsingular for $S K>3 \sum_{j=1}^{S} q_{j}+p+S$, so $\mathbf{J}_{K}^{-1}\left(\boldsymbol{\theta}_{M}^{0}\right)$ exists. Lemma 5 provides the blocks of $\lim _{K \rightarrow \infty} \mathbf{J}_{K}\left(\boldsymbol{\theta}_{M}^{0}\right)$. Likewise, Lemma 6 defines the diagonal blocks of the variance component $\mathbf{J}^{V}\left(\boldsymbol{\theta}_{V}^{0}\right)=\operatorname{diag}\left(\mathbf{J}^{V}\left(\boldsymbol{\theta}_{1}^{(v) 0}\right), \ldots, \mathbf{J}^{V}\left(\boldsymbol{\theta}_{S}^{(v) 0}\right)\right)$ which are also positive definite. Putting these together defines $\mathbf{J}\left(\boldsymbol{\theta}^{0}\right)=\operatorname{diag}\left(\mathbf{J}_{M}\left(\boldsymbol{\theta}_{M}^{0}\right)\right.$, $\left.\mathbf{J}_{V}\left(\boldsymbol{\theta}_{V}^{0}\right)\right)$.

(v) $\operatorname{Esup}_{\boldsymbol{\theta} \in \mathcal{N}}\left\|\frac{\partial^{2}}{\partial \boldsymbol{\theta} \partial \boldsymbol{\theta}^{\prime}} \ln f(z \mid \boldsymbol{\theta})\right\|<\infty$. Lemmas 7 and 8 show that the deterministic components in the matrix $\frac{\partial^{2}}{\partial \boldsymbol{\theta} \partial \boldsymbol{\theta}^{\prime}} \ln f(\varepsilon \mid \boldsymbol{\theta})$ are bounded for $\boldsymbol{\theta} \in \mathcal{N}$. Then the expectation of the supremum is bounded as well.

Finally, since matrix inversion is a continuous transformation, it follows from the continuous mapping theorem that when $\mathbf{J}_{K}\left(\boldsymbol{\theta}^{0}\right) \rightarrow \mathbf{J}\left(\boldsymbol{\theta}^{0}\right)$, then $\mathbf{J}_{K}^{-1}\left(\boldsymbol{\theta}^{0}\right) \rightarrow \mathbf{J}^{-1}\left(\boldsymbol{\theta}^{0}\right)$. This concludes the proof.

Proof of Lemma 11. First consider

$$
\mathbf{J}_{K}^{A}\left(\boldsymbol{\alpha}_{s}\right)=\mathrm{E} s_{K}\left(\boldsymbol{\alpha}_{s}\right) s_{K}^{\prime}\left(\boldsymbol{\alpha}_{s}\right)=\frac{1}{\sigma^{2} K} \sum_{k=0}^{K-1} \mathbf{k}_{s k+s} \mathbf{k}_{s k+s}^{\prime} .
$$


Letting $k=[r K]$ in (39) yields

$$
\begin{aligned}
\mathbf{J}_{K}^{A}\left(\boldsymbol{\alpha}_{s}\right) & =\frac{1}{\sigma^{2}} \sum_{k=0}^{K-1} \int_{\frac{S k+s}{S K}}^{\frac{S(k+1)+s}{S K}} \mathbf{k}_{s[r K]+s} \mathbf{k}_{s[r K]+s}^{\prime} \mathrm{d} r \\
& =\frac{1}{\sigma^{2}} \int_{\frac{s}{S K}}^{\frac{S K+s}{S K}} \mathbf{k}_{s[r K]+s} \mathbf{k}_{s[r K]+s}^{\prime} \mathrm{d} r \\
& \rightarrow \frac{1}{\sigma^{2}} \int_{0}^{1} \mathbf{r r}^{\prime} \mathrm{d} r=\frac{1}{\sigma^{2}}\left[\begin{array}{rrr}
1 / 3 & 1 / 4 & 1 / 5 \\
& 1 / 5 & 1 / 6 \\
& & 1 / 7
\end{array}\right]=\mathbf{J}^{A}\left(\boldsymbol{\alpha}_{s}\right)
\end{aligned}
$$

for $s=1, \ldots, S$. Next, using the same arguments,

$$
\begin{aligned}
\mathbf{J}_{K}^{A}\left(\boldsymbol{\alpha}_{s}, \alpha_{s 0}\right) & =\frac{1}{\sigma^{2} K} \sum_{k=0}^{K-1} \mathbf{k}_{s k+s} \rightarrow \frac{1}{\sigma^{2}} \int_{0}^{1} \mathbf{r} \mathrm{d} r \\
& =\frac{1}{\sigma^{2}}\left(\frac{1}{2}, \frac{1}{3}, \frac{1}{4}\right)^{\prime}=\mathbf{J}^{A}\left(\boldsymbol{\alpha}_{s}, \alpha_{s 0}\right)
\end{aligned}
$$

as $K \rightarrow \infty$. Following the same line of reasoning and assuming that A5 holds,

$$
\begin{aligned}
\mathbf{J}_{S K}^{A}\left(\alpha_{s 0}, \phi^{0}\right) & =\frac{1}{\sigma^{2} K} \sum_{k=0}^{K-1} \mathrm{Ey}_{S k+s-1}^{\prime} \\
& =\frac{1}{\sigma^{2} \phi(1) K} \sum_{k=0}^{K-1}\left\{\delta_{s-1}\left(\frac{S k+s-1}{S K}\right), \ldots, \delta_{s-p}\left(\frac{S k+s-p}{S K}\right)\right) \\
& \rightarrow \frac{1}{\sigma^{2} \phi(1)} \int_{0}^{1} \boldsymbol{\delta}_{s-1}^{\prime}(r) \mathrm{d} r=\mathbf{J}^{A}\left(\alpha_{s 0}, \phi^{0}\right)
\end{aligned}
$$

and

$$
\mathbf{J}_{S K}^{A}\left(\boldsymbol{\alpha}_{s}, \boldsymbol{\phi}\right)=\frac{1}{\sigma^{2} K} \sum_{k=0}^{K-1} \boldsymbol{\alpha}_{s} \mathrm{Ey}_{S k+s-1}^{\prime} \rightarrow \frac{1}{\sigma^{2} \phi(1)} \int_{0}^{1} \mathbf{r} \boldsymbol{\delta}_{s-1}^{\prime}(r) \mathrm{d} r=\mathbf{J}^{A}\left(\boldsymbol{\alpha}_{s}, \boldsymbol{\phi}\right)
$$

as $K \rightarrow \infty$.

Proof of Lemma 12. We only have to prove (32). We have

$$
\mathrm{Es}_{k}\left(\boldsymbol{\alpha}_{s}\right) \mathbf{s}_{k}^{\prime}\left(\boldsymbol{\theta}_{s}^{0}\right)=\frac{1}{\sigma^{2} K} \sum_{k=0}^{K-1} \mathbf{k}_{S k+s} \frac{\partial \delta_{s}\left(\frac{S k+s}{S K}\right)}{\partial \boldsymbol{\theta}_{s}^{\prime}}
$$


Setting $k=[r K]$ in (40) yields

$$
\begin{aligned}
\mathrm{Es}_{k}\left(\boldsymbol{\alpha}_{s}\right) \mathbf{s}_{k}^{\prime}\left(\boldsymbol{\theta}_{s}^{0}\right) & =\frac{1}{\sigma^{2}} \sum_{k=0}^{K-1} \int_{\frac{S k+s}{S K}}^{\frac{S(k+1)+s}{S K}} \mathbf{k}_{S[r K]+s} \frac{\partial \delta_{s}\left(\frac{S[r K]+s}{S K}\right)}{\partial \boldsymbol{\theta}_{s}^{\prime}} \mathrm{d} r \\
& =\frac{1}{\sigma^{2}} \int_{\frac{s}{S K}}^{\frac{S K+s}{S K}} \mathbf{k}_{S[r K]+s} \frac{\partial \delta_{s}\left(\frac{S[r K]+s}{S K}\right)}{\partial \boldsymbol{\theta}_{s}^{\prime}} \mathrm{d} r \\
& \rightarrow \frac{1}{\sigma^{2}} \int_{0}^{1} \mathbf{r} \frac{\partial \delta_{s}(r)}{\partial \boldsymbol{\theta}_{s}^{\prime}} \mathrm{d} r=\mathbf{J}^{A}\left(\boldsymbol{\alpha}_{s}, \boldsymbol{\theta}_{s}^{0}\right)
\end{aligned}
$$

which is the desired result.

\section{B Testing the constancy of error variance month by month}

Constancy of error variances is tested as in Silvennoinen and Teräsvirta (2016). The difference is that because of seasonal heteroskedasticity, the test is conducted separately for each month. It has been argued, see for example van Dijk, Franses and Lucas (1999), that additive outliers may bias the test of no ARCH towards rejection. Since this may also be the case here, we augment the test statistic by impulse dummy variables $\bar{D}_{S k+s}^{\left(t_{q}\right)}: \bar{D}_{S k+s}^{\left(t_{q}\right)}=1$ for $S k+s=t_{q}$, zero otherwise. The test in the $T R^{2}$ form is conducted as follows:

1. Form the log-likelihood of the auxiliary model for season $s$ ( $s$ is fixed, there are $K$ observations). It equals

$$
L_{K}^{(s)}=c-\frac{1}{2} \sum_{k=0}^{K-1} \ln \sigma_{S k+s}^{2}-\frac{1}{2} \sum_{k=0}^{K-1} \frac{\widehat{\varepsilon}_{S k+s}^{2}}{\sigma_{S k+s}^{2}}
$$

where the observations are $s, 2 s, \ldots,(K-1) s$. For each season $s=$ $1, \ldots, S$, there is a separate error variance

$$
\sigma_{S k+s}^{2}=\alpha_{s 0}+\sum_{q=1}^{Q_{s}} \alpha_{i}^{D} \bar{D}_{S k+s}^{\left(t_{q}\right)}+\alpha_{s 1} \frac{k+1}{K}+\alpha_{s 2}\left(\frac{k+1}{K}\right)^{2}+\alpha_{s 3}\left(\frac{k+1}{K}\right)^{3}
$$

for $k=0,1, \ldots, K-1$. Estimate the auxiliary variance model by maximising $L_{K}^{(s)}$ under $\mathrm{H}_{0}: \alpha_{s 1}=\alpha_{s 2}=\alpha_{s 3}=0$. Save the standardised squared residuals

$$
\widehat{\phi}_{k+s}^{2}=\frac{\widehat{\varepsilon}_{S k+s}^{2}}{\widehat{\alpha}_{s 0}^{2}+\sum_{q=1}^{Q} \widehat{\alpha}_{i}^{D} \bar{D}_{S k+s}^{\left(t_{q}\right)}}
$$


and compute the 'residual sum of squares' $S S R_{0}=\sum_{k=0}^{K-1}\left(\widehat{\phi}_{S k+s}^{2}-1\right)^{2}$.

2. Regress $\left(\phi_{S k+s}^{2}-1\right)^{2}$ on $1, \bar{D}_{S k+s}^{\left(t_{q}\right)}, q=1, \ldots, Q_{s}, \frac{k+1}{K},\left(\frac{k+1}{K}\right)^{2}$ and $\left(\frac{k+1}{K}\right)^{3}$ and form the sum of squared residuals $S S R_{1}$.

3. Calculate the test statistic

$$
L M_{s}=K \frac{S S R_{0}-S S R_{1}}{S S R_{0}} .
$$

It has an asymptotic $\chi^{2}$-distribution with three degrees of freedom when $\mathrm{H}_{0}$ holds. As already mentioned, the test is carried out for each $s$ separately, because the seasonal error variables are orthogonal. 


\section{Estimated equations}

\begin{tabular}{|c|c|c|c|}
\hline & Estimate & Std. Error & $\mathrm{t}$ value \\
\hline$\phi_{1}$ & 0.235 & 0.018 & 12.8 \\
\hline$\phi_{2}$ & 0.057 & 0.018 & 3.08 \\
\hline$\delta_{1,0}$ & 1.29 & 0.204 & 6.31 \\
\hline$\delta_{1,1}$ & 1.18 & 0.202 & 5.84 \\
\hline$c_{1}$ & 0.315 & 0.031 & \\
\hline$\delta_{2,0}$ & 3.02 & 0.122 & 24.8 \\
\hline$\delta_{3,0}$ & 4.09 & 0.166 & 24.6 \\
\hline$\delta_{3,1}$ & 1.19 & 0.758 & 1.57 \\
\hline$\gamma_{3}$ & 10.1 & 11.1 & \\
\hline$c_{3}$ & 0.751 & 0.155 & \\
\hline$\delta_{4,0}$ & 6.56 & 0.139 & 47.3 \\
\hline$\delta_{5,0}$ & 9.10 & 0.178 & 51.1 \\
\hline$\delta_{6,0}$ & 11.2 & 0.236 & 47.6 \\
\hline$\delta_{7,0}$ & 11.9 & 0.304 & 39.2 \\
\hline$\delta_{7,1}$ & 0.758 & 0.296 & 2.56 \\
\hline$c_{7}$ & 0.845 & 0.053 & \\
\hline$\delta_{8,0}$ & 11.0 & 0.351 & 31.3 \\
\hline$\delta_{8,1}$ & 0.725 & 0.398 & 1.82 \\
\hline$\gamma_{8}$ & 35.0 & 68.0 & \\
\hline$c_{8}$ & 0.848 & 0.067 & \\
\hline$\delta_{9,0}$ & 8.60 & 0.381 & 22.6 \\
\hline$\delta_{9,1}$ & 1.89 & 0.679 & 2.79 \\
\hline$\gamma_{9}$ & 6.45 & 4.25 & \\
\hline$\delta_{10,0}$ & 5.54 & 0.340 & 16.3 \\
\hline$\delta_{10,1}$ & 1.11 & 0.295 & 3.77 \\
\hline$\gamma_{10}$ & 21.6 & 21.3 & \\
\hline$c_{10}$ & 0.722 & 0.053 & \\
\hline$\delta_{11,0}$ & 2.36 & 0.684 & 3.46 \\
\hline$\delta_{11,1}$ & 3.19 & 1.083 & 2.94 \\
\hline$\gamma_{11}$ & 2.73 & 2.03 & \\
\hline$\delta_{12,0}$ & 1.48 & 0.291 & 5.09 \\
\hline$\delta_{12,1}$ & 0.927 & 0.250 & 3.70 \\
\hline$c_{12}$ & 0.203 & 0.042 & \\
\hline SSR & \multicolumn{3}{|c|}{5302} \\
\hline log.Lik & \multicolumn{3}{|c|}{$-5036(\mathrm{df}=34)$} \\
\hline
\end{tabular}

The cases (January, July, December) in which $\gamma=40$ (fixed), this value is not shown in the table. The same is true for cases (September, November) in which $c=1$.

No $t$-values are reported for the estimates of $\gamma$ and $c$. For $\widehat{c}$, significant deviations from zero are of no interest. As to $\widehat{\gamma}$, due to the identification problem the asymptotic distribution of the $t$-statistic is unknown, and reporting a $t$-value is therefore misleading.

Table 7: Estimates of the mean component of the SSM-AR model for the monthly CET series, 1772-2016 


\section{Additional tables}

\begin{tabular}{rrrr}
\hline & 1 & 2 & 3 \\
\hline Jan & 0.4584 & 0.7407 & 0.6706 \\
Feb & 0.8951 & 0.6911 & 0.7777 \\
Mar & 0.2604 & 0.3469 & 0.2236 \\
Apr & 0.0831 & 0.0302 & 0.0703 \\
May & 0.0486 & 0.1331 & 0.2433 \\
Jun & 0.1586 & 0.3681 & 0.5723 \\
Jul & 0.9711 & 0.9792 & 0.9299 \\
Aug & 0.7816 & 0.9410 & 0.9673 \\
Sep & 0.9340 & 0.5550 & 0.6793 \\
Oct & 0.5983 & 0.8135 & 0.7642 \\
Nov & 0.9494 & 0.4363 & 0.4173 \\
Dec & 0.8028 & 0.8245 & 0.2504 \\
\hline
\end{tabular}

Table 8: $p$-values of tests of constancy of the error variance based on the first, second and third order error variance. The errors outside the \pm 3 standard error band are treated as outliers and dummied out 
2017-37: $\quad$ Søren Johansen and Morten Ørregaard Nielsen: Testing the CVAR in the fractional CVAR model

2017-38: Nektarios Aslanidis and Charlotte Christiansen: Flight to Safety from European Stock Markets

2017-39: Tommaso Proietti, Niels Haldrup and Oskar Knapik: Spikes and memory in (Nord Pool) electricity price spot prices

2018-01: Emilio Zanetti Chini: Forecaster's utility and forecasts coherence

2018-02: Torben G. Andersen, Nicola Fusari and Viktor Todorov: The Pricing of Tail Risk and the Equity Premium: Evidence from International Option Markets

2018-03: Torben G. Andersen, Nicola Fusari, Viktor Todorov and Rasmus T. Varneskov: Unified Inference for Nonlinear Factor Models from Panels with Fixed and Large Time Span

2018-04: $\quad$ Torben G. Andersen, Nicola Fusari, Viktor Todorov and Rasmus T. Varneskov: Option Panels in Pure-Jump Settings

2018-05: $\quad$ Torben G. Andersen, Martin Thyrsgaard and Viktor Todorov: Time-Varying Periodicity in Intraday Volatility

2018-06: Niels Haldrup and Carsten P. T. Rosenskjold: A Parametric Factor Model of the Term Structure of Mortality

2018-07: $\quad$ Torben G. Andersen, Nicola Fusari and Viktor Todorov: The Risk Premia Embedded in Index Options

2018-08: $\quad$ Torben G. Andersen, Nicola Fusari and Viktor Todorov: Short-Term Market Risks Implied by Weekly Options

2018-09: $\quad$ Torben G. Andersen and Rasmus T. Varneskov: Consistent Inference for Predictive Regressions in Persistent VAR Economies

2018-10: Isabel Casas, Xiuping Mao and Helena Veiga: Reexamining financial and economic predictability with new estimators of realized variance and variance risk premium

2018-11: $\quad$ Yunus Emre Ergemen and Carlos Velasco: Persistence Heterogeneity Testing in Panels with Interactive Fixed Effects

2018-12: $\quad$ Hossein Asgharian, Charlotte Christiansen and Ai Jun Hou: Economic Policy Uncertainty and Long-Run Stock Market Volatility and Correlation

2018-13: Emilio Zanetti Chini: Forecasting dynamically asymmetric fluctuations of the U.S. business cycle

2018-14: Cristina Amado, Annastiina Silvennoinen and Timo Teräsvirta: Models with Multiplicative Decomposition of Conditional Variances and Correlations

2018-15: Changli He, Jian Kang, Timo Teräsvirta and Shuhua Zhang: The Shifting Seasonal Mean Autoregressive Model and Seasonality in the Central England Monthly Temperature Series, 1772-2016 